\title{
Non-homogeneous dispersion of graphene in polyacrylonitrile substrates induces a migrastatic response and epithelial-like differentiation in MCF7 breast cancer cells
}

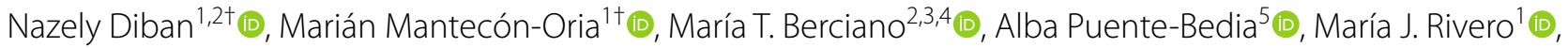 \\ Ane Urtiaga ${ }^{1,2}$, Miguel Lafarga ${ }^{2,3,6^{*}}$ (D) and Olga Tapia ${ }^{2,3,7^{*}}$ (D)
}

\begin{abstract}
${ }^{*}$ Correspondence:
lafargam@unican.es; olga.

tapia@uneatlantico.es

†Nazely Diban and Marián

Mantecón-Oria contributed equally to this work

${ }^{6}$ Department of Anatomy and Cell Biology, University

of Cantabria, Santander,

Spain

7 "Universidad Europea del Atlántico", Santander, Spain Full list of author information is available at the end of the article
\end{abstract}

\begin{abstract}
Background: Recent advances from studies of graphene and graphene-based derivatives have highlighted the great potential of these nanomaterials as migrastatic agents with the ability to modulate tumor microenvironments. Nevertheless, the administration of graphene nanomaterials in suspensions in vivo is controversial. As an alternative approach, herein, we report the immobilization of high concentrations of graphene nanoplatelets in polyacrylonitrile film substrates (named PAN/G10) and evaluate their potential use as migrastatic agents on cancer cells.
\end{abstract}

Results: Breast cancer MCF7 cells cultured on PAN/G10 substrates presented features resembling mesenchymal-to-epithelial transition, e.g., (i) inhibition of migratory activity; (ii) activation of the expression of E-cadherin, cytokeratin 18, ZO-1 and EpCAM, four key molecular markers of epithelial differentiation; (iii) formation of adherens junctions with clustering and adhesion of cancer cells in aggregates or islets, and (iv) reorganization of the actin cytoskeleton resulting in a polygonal cell shape. Remarkably, assessment with Raman spectroscopy revealed that the above-mentioned events were produced when MCF7 cells were preferentially located on top of graphene-rich regions of the PAN/G10 substrates.

Conclusions: The present data demonstrate the capacity of these composite substrates to induce an epithelial-like differentiation in MCF7 breast cancer cells, resulting in a migrastatic effect without any chemical agent-mediated signaling. Future works will aim to thoroughly evaluate the mechanisms of how PAN/G10 substrates trigger these responses in cancer cells and their potential use as antimetastatics for the treatment of solid cancers.

Keywords: Graphene, Polyacrylonitrile, Mesenchymal-to-epithelial transition (MET), Migrastatic agent, Cancer cells

(c) The Author(s), 2021. Open Access This article is licensed under a Creative Commons Attribution 4.0 International License, which permits use, sharing, adaptation, distribution and reproduction in any medium or format, as long as you give appropriate credit to the original author(s) and the source, provide a link to the Creative Commons licence, and indicate if changes were made. The images or other third party material in this article are included in the article's Creative Commons licence, unless indicated otherwise in a credit line to the material. If material is not included in the article's Creative Commons licence and your intended use is not permitted by statutory regulation or exceeds the permitted use, you will need to obtain permission directly from the copyright holder. To view a copy of this licence, visit http:// creativecommons.org/licenses/by/4.0/. The Creative Commons Public Domain Dedication waiver (http://creativecommons.org/publi cdomain/zero/1.0/) applies to the data made available in this article, unless otherwise stated in a credit line to the data. 


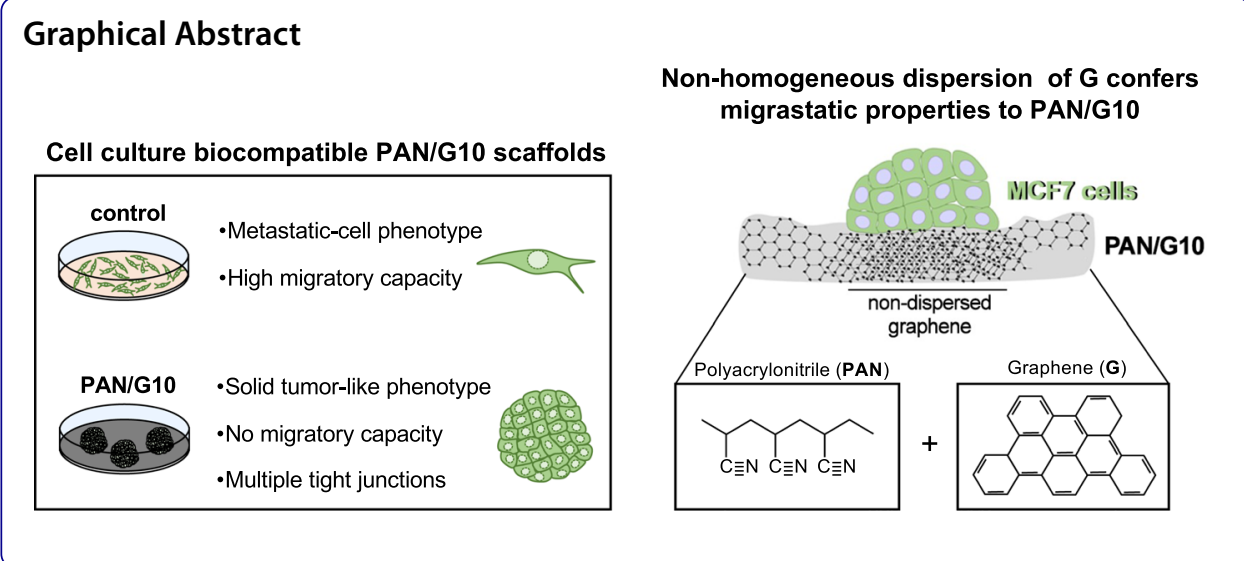

\section{Background}

Solid cancers are characterized by a dysregulation of cellular proliferation and the metastatic potential of cancer cells derived from the primary tumor to invade and spread to other organs of the body. Abnormal cellular proliferation of cancer cells is dependent on dysregulation of the cell cycle, especially of the G1-S phase transition, produced by the altered expression of oncogenes (upregulation) and tumor suppressor genes (downregulation) implicated in cell cycle control (Weinberg 2014). In solid cancers, different mechanisms are involved in the ability of cancer cells to migrate and invade through the extracellular matrix to establish metastatic secondary tumors. They include: (i) the loss of key epithelial markers, most notably E-cadherin of intercellular adherens junctions as a result of downregulation or inactivation of E-cadherin gene ( $C D H 1)$ expression; (ii) the altered expression of integrins that destabilizes the cell-cellular matrix interactions; (iii) the enhanced production of extracellular matrix (ECM)-degrading enzymes, such as metalloproteases, that facilitates amoeboid migration of cancer cells through the extracellular matrix, and (iv) cytoskeletal reorganization including replacement of cytokeratins by vimentin and the Rho/Rho kinase (ROCK) pathway-dependent dysregulation of actin dynamics, which impact actin polymerization and contractility (Craene and Berx 2013; Hanahan and Weinberg 2011; Jung et al. 2020). Importantly, the latter mechanism is required for cancer cell migration and invasion. Indeed, cancer cells undergo changes in both cell shape, involving the formation of actin-based protrusions and invadosomes, and actomyosin contractility, which is required for rear retraction and translocation of tumor cell bodies (Kümper and Marshall 2011; Masi et al. 2020; Sanz-Moreno et al. 2011).

Based on the properties of cancer cells, two main strategic approaches are used in the treatment of solid cancers: antiproliferative approaches directed at eliminating rapidly proliferating cancer cells and approaches focused on cellular processes related to the ability of malignant tumor cells to migrate, invade and metastasize. Importantly, more than $90 \%$ of morbidity and mortality in solid cancer is based on invasion and metastasis (Gandalovičová et al. 2017; Sleeman and Steeg 2010). In this context, great efforts have been made by medicinal chemists to design new antiproliferative agents that produce tumor reductions, a basic requirement for their approval as cancer drugs. Similarly, "migrastatics" is a recently coined term for drugs that interfere 
with the ability of cancer cells to migrate and metastasize (Gandalovičová et al. 2017). New migrastatic drugs are increasingly being tested and may provide new therapeutic options for solid cancers. At present, the most promising drugs are inhibitors of the ROCK/MRCK or ROCK/PKA/PKB kinases (Gandalovičová et al. 2017).

Cancer nanotheranostics (nanotechnology for the diagnosis and therapy of cancer) has been promoted as a promising alternative to treat cancer stem cells (CSCs), which cause most cancer recurrences and are especially resistant to radiotherapies and chemotherapies (Misra et al. 2020). In particular, in recent years, graphene and graphene derivatives, a group of nanomaterials with remarkable physicochemical properties and biological compatibility, have shown great promise as effective treatments of glioblastomas (Martelli et al. 2020). Furthermore, graphene and graphene oxide displayed migrastatic properties in cancer cells when these compounds were applied as nanomaterial suspensions by impairing normal mitochondrial activity and the ATP cycle (Zhou et al. 2014).

Graphene-based nanomaterials can enter cells via cell transduction. Once inside the cell, their molecular mechanisms are triggered. Moreover, nanomaterial-based anticancer treatments have demonstrated effective mechanisms to modulate tumor microenvironments with characteristics including enhanced permeability and retention (EPR), acidosis, extensive angiogenesis, and tumor-associated immune cells (Saleem et al. 2018). Nevertheless, there is still pointed controversy about the most effective mode of administration of graphene nanomaterials, the impact of administration route on the fate of the nanomaterials in vivo and their possible side effects.

Over the last two decades, there has been a growing interest in tissue engineering for developing polymer-based membranes capable of modulating the function and fate of cells, including proliferation, adhesion, migration and differentiation, by tuning, i.e., the cell substrate topographical/mechanical microenvironment and chemical cell-substrate interactions (Carré and Lacarrière 2010; Downing et al. 2013). Different graphene-polymer composites, i.e., composite membranes of poly( $\varepsilon$-caprolactone)/graphene-based nanomaterials ( $\mathrm{PCL} / \mathrm{GbN})$, have been demonstrated to induce neural differentiation (Sánchez-González et al. 2018) and provided promising results to develop useful experimental models for in vitro studies of cell behavior in different biological systems (Mantecón-Oria et al. 2020). For instance, polymer-based membranes may be used to analyze cellular mechanisms involved in neural differentiation, organization of the blood-brain barrier and cancer. It has been previously reported that somatic fibroblasts were efficiently reprogrammed into induced pluripotent stem cells (iPSCs) in a process similar to a mesenchymal-to-epithelial-transition (MET) when grown on graphene-based substrates (Yoo et al. 2014). Therefore, we hypothesized that a graphene-immobilized polymer membrane could be used as a migrastatic substrate alternative to the administration of suspended nanoparticles for cancer therapy.

In this study, we used the biocompatible polymer polyacrylonitrile (PAN) to immobilize between 2 and 15\% w/w of graphene (G) by synthesizing PAN/G membranes as scaffolds for breast cancer MCF7 cells in culture. This cell line is widely used as in vitro models in research for estrogen receptor (ER)-positive breast cancer cell experiments (Comşa et al. 2015). As estrogen (E2)-sensitive cells, MCF7 expresses significant levels of ER $\alpha$ transcripts (Brooks et al. 1973). The main aim is to investigate whether the presence of immobilized graphene in polymer substrates can reverse some cancer cell 
properties such as loss of adhesion, migration and invasiveness, and to induce differentiation toward an epithelial cell-like phenotype. The present work evaluates the contribution of PAN/G membranes to promote a phenotypic change in breast cancer MCF7 cells, involving the (i) inhibition of migratory activity; (ii) upregulation of key epithelial markers with the formation of adherens junctions and cellular aggregation in islets, and (iii) reorganization of the actin cytoskeleton resulting in a polygonal cell shape. Thus, PAN/G biomaterials enriched in graphene acting as a migrastatic agent (Gandalovičová et al. 2017) may provide a new option for in vitro trials of solid cancer therapy. Moreover, the migrastatic anti-invasion properties of these biomaterials open a new door for their potential use as tissue implants in animal models of cancer.

\section{Results}

\section{Morphology of the membranes}

Figure 1 presents representative SEM images of the surface (Fig. $1 \mathrm{~A}-\mathrm{H}$ ) and crosssection (Fig. 1I-L) of the PAN and PAN/G membranes. All PAN/G membranes present the typical morphology with a dense (nonporous) surface and finger-like pore structures in the cross-section characteristic of a fast phase inversion between the solvent and coagulant during membrane synthesis. The progressive incorporation of graphene nanomaterials altered the phase inversion thermodynamics and kinetics of the ternary system and acted as a point of rupture during the polymer coagulation process, distorting the neat finger-like structure of the cross-section, particularly at higher concentrations $(10 \%$ and $15 \% \mathrm{w} / \mathrm{w})$ (Fig. $1 \mathrm{~K}, \mathrm{~L})$ and producing rough top surfaces and cracks (Fig. 1B-D, F-H) (Romay et al. 2020). The cracks and the fraction of porosity, or pore fraction (PF), on the top surface were maximal in PAN/G2 membranes and then progressively decreased in PAN/G10 and PAN/G15 membranes (Fig. 1F-H; Table 1). The cross-sectional pore fraction was significantly increased for the PAN/G15 membranes relative to the other membranes (Table 1).

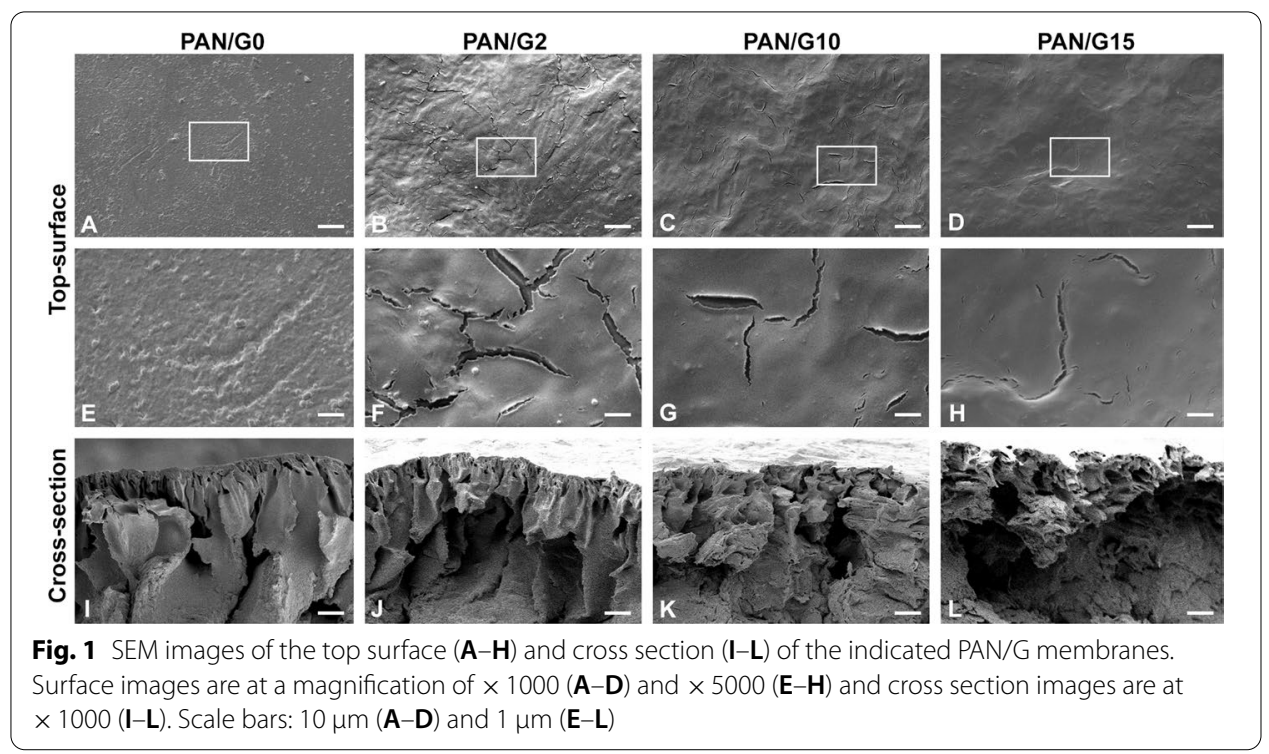


Table 1 Thickness and pore fraction (PF) in the top-surface and in the cross section of the PAN/G membranes

\begin{tabular}{llll}
\hline Membrane & Thickness $(\boldsymbol{\mu m})$ & Top-surface PF $(\%)$ & Cross section PF (\%) \\
\hline PAN/G0 & $249 \pm 21$ & $0.2 \pm 0.1$ & $15.1 \pm 3.3$ \\
PAN/G2 & $187 \pm 9$ & $1.8 \pm 0.9$ & $24.9 \pm 11.5$ \\
PAN/G10 & $203 \pm 5$ & $1.7 \pm 0.6$ & $17.7 \pm 2.5$ \\
PAN/G15 & $196 \pm 15$ & $0.1 \pm 0.1$ & $47.9 \pm 10.7^{*}$ \\
\hline
\end{tabular}

Statistical significance respect to PAN/G0 ( $\left.{ }^{*} p<0.05\right)$

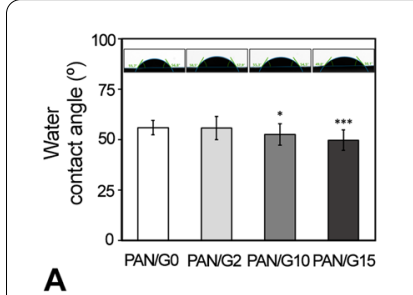

A
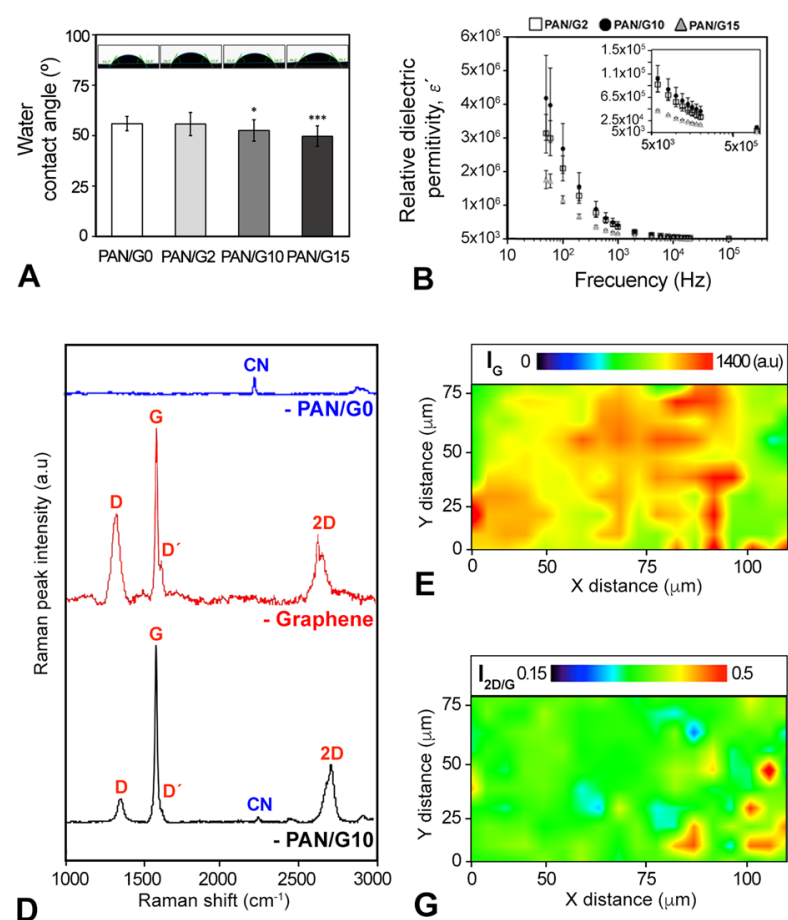

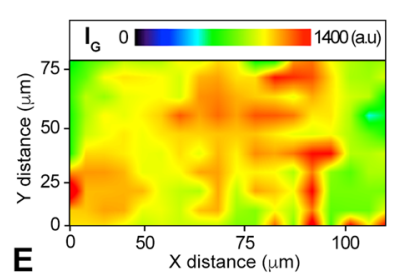

E

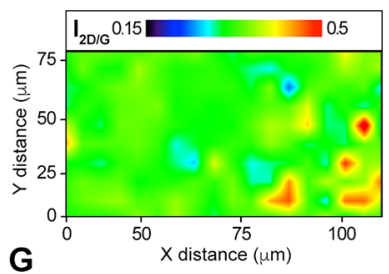

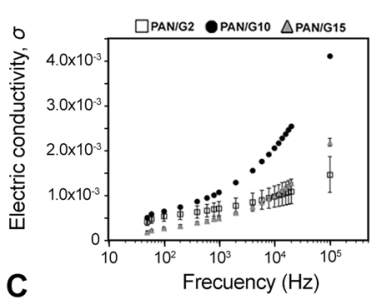

C
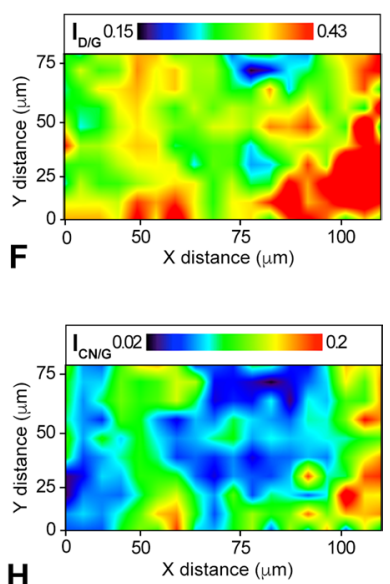

Fig. 2 A Water contact angle average values of the indicated PAN/G membranes. B, C Plots of the relative dielectric permittivity $(\mathbf{B})$ and electric conductivity $(\mathbf{C})$ at different values of applied frequency of the PAN/ G2; PAN/G10 and PAN/G15 membranes measured by means of electrical impedance. D Comparison of the Raman spectra of graphene (red), PAN/G0 (blue) and PAN/G10 membranes (black). E-H Surface mapping $(0.144 \times 0.088 \mathrm{~mm})$ of PAN/G10 membrane showing the intensity of band $\mathrm{G}$ of graphene $\left(I_{G}, \mathbf{E}\right)$, intensity ratio of $D$ and $G$ bands of graphene $\left(I_{\mathrm{D} / \mathrm{G}}, \mathbf{F}\right)$ intensity ratio of $2 \mathrm{D}$ and $\mathrm{G}$ bands graphene $\left(I_{2 \mathrm{D} / \mathrm{G}}, \mathbf{G}\right)$ and intensity ratio of nitrile $(\mathrm{CN})$ characteristic band of PAN polymer and $\mathrm{G}$ band of graphene $\left(I_{\mathrm{CN} / \mathrm{G}} \mathbf{H}\right)$

\section{Water contact angle, electrical properties and graphene distribution of PAN/G membranes}

Figure 2A shows the water contact angle (WCA) of the PAN/G membranes. The WCA values were below $60^{\circ}$ in all substrates, indicating the overall hydrophilic nature of the membranes. The presence of graphene affected the WCA progressively decreasing from $56^{\circ} \pm 4^{\circ}$ for PAN $/ G 0$ to $56^{\circ} \pm 6^{\circ}$ for PAN/G2, to $53^{\circ} \pm 5^{\circ}$ for PAN/G10 and to $50^{\circ} \pm 5^{\circ}$ for PAN/G15 membranes. Although graphene has been traditionally considered a hydrophobic material, recent studies (Prydatko et al. 2018) demonstrated the hydrophilic nature of single- and multi-layered graphene (WCA c.a. $42^{\circ} \pm 3^{\circ}$ ). The contact angle of PAN/G membrane substrates using DMEM culture medium also decreased progressively, although more drastically, as the load of graphene increased from $61^{\circ} \pm 7^{\circ}$ for 
PAN/G0 membranes down to $35^{\circ} \pm 5^{\circ}$ (see Additional file 1: Fig. S1A). Due to the polar nature of culture medium, usually lower contact angles have been reported with this fluid (Charles-Harris et al. 2005).

Figure 2B, C shows the relative dielectric permittivity and electrical conductivity of the different PAN/G membranes at different graphene doping concentrations $(2 \%, 10 \%$ and $15 \% \mathrm{w} / \mathrm{w}$ ). Electrical impedance of PAN/G membranes wet with culture medium was also measured (Additional file 1: Fig. S1B, C) revealing that the presence of ions in the culture medium increased 2 orders of magnitude the electrical conductivity and permittivity of the dried membrane samples. There were no evident differences among the PAN/G membranes electrical properties, particularly on the permittivity. Therefore, the presence of culture medium might mask the influence of the effect of graphene loading into the intrinsic electric properties of the PAN/G membranes.

In agreement with the electrical properties of the other polymer-graphene composites [i.e., PVDF-graphene (Xia et al. 2017)], the results for the PAN/G membranes (Fig. 2B, C) showed that for all graphene loadings, the conductivity increased with a frequency attributed to a frequency-assisted electron hopping effect that occurs when the frequency increases. In parallel, the dielectric permittivity decreased with the frequency.

At low frequencies, the relative dielectric permittivity reached outstandingly high values on the order of $10^{6}$, similar to the values found for PVDF-graphene composites at graphene loadings above the percolation threshold concentration. The percolation threshold is the minimum loading of conductive materials (graphene nanoplatelets in this case) in the polymer composite that allows for sufficient proximity between the fillers to facilitate electron transfer in the material. This result could be associated with the so-called Maxwell-Wagner-Sillars (MWS) interfacial polarization effect; that is, at low frequencies, a slow change in the electric field provides enough time for the permanent and induced dipoles to align themselves. Nonetheless, at high frequencies, the electrons accumulated at the interface from the MWS polarization mechanism decrease, causing a progressive reduction of dielectric permittivity with an increased frequency. According to these results, the percentage of graphene incorporated into the PAN/G membranes reported here would be above the percolation value.

The results show that PAN/G10 membranes have the maximal electrical conductivity and dielectric permittivity, while the PAN/G15 membranes surprisingly have a lower conductivity (below $10^{4} \mathrm{~Hz}$ ) and dielectric permittivity (over the whole frequency range studied) than the PAN/G2 membranes. This reduction in electrical properties for high loadings of graphene nanoparticles in polymer matrices was previously attributed to the formation of large graphene aggregates that handicapped charge transport networks (Xia et al. 2017). Another possible explanation might be the larger voids or pores found in the cross-section of PAN/G15 membranes not found in the other membranes (Fig. 1L). Other works also reported a reduction in the electrical properties of ceramic and polymer materials with increasing porosity of the substrates (El Khal et al. 2017; Sarafis and Nassiopoulou 2014).

Several studies evaluating the electrical impedance of breast cancer tissues and cell suspensions have shown their higher electrical conductivity and permittivity compared to normal breast cells and tissues (Surowiec et al. 1988; Wang et al. 2021). A significant correlation between migration rate and electrical conductivity and permittivity of breast 
cancer cell suspensions was observed previously by Wang et al. (2021). Moreover, they also observed a clear influence of microenvironment on the electrical properties of the breast cancer cells and cell suspensions. Since cells are continuously exchanging material (energy and biomolecules) with the surrounding, the microenvironment electrical properties can be assumed to be intimately connected with electrical properties of cells tissues at cellular level. Overall, these results show that the PAN/G10 membranes presented optimum electrical properties.

Figure 2D shows the characteristic Raman spectra for the PAN/G0 membranes, commercial graphene nanoplatelets and PAN/G10 membranes. The spectrum of commercial graphene presented four relevant peaks: the D band at $1322 \mathrm{~cm}^{-1}$ was associated with the presence of carbon atoms with $\mathrm{sp}^{3}$ hybridization or structural disorders in the carbon network, the $\mathrm{G}$ band at $1583 \mathrm{~cm}^{-1}$ was attributed to the first-order scattering of the $E_{2 g}$ phonon of $\mathrm{sp}^{2}$ carbon atoms, the $\mathrm{D}^{\prime}$ band at $1615 \mathrm{~cm}^{-1}$ was superimposed with the $\mathrm{G}$ band and was related to edge defects, and the $2 \mathrm{D}$ band at $2635 \mathrm{~cm}^{-1}$ was associated with the multi-layered character of graphene (Kim et al. 2017; Mantecón-Oria et al. 2020) (Fig. 2D, red spectrum). The $I_{2 \mathrm{D}} / I_{\mathrm{G}}$ ratio of 0.4 confirmed that the nanomaterial had 6-7 layers, as indicated by the supplier (Avanzare).

The PAN/G0 spectrum includes a band typical of nitrile groups $(-\mathrm{CN})$ at $2245 \mathrm{~cm}^{-1}$ (Fig. 2D, blue spectrum). The Raman spectrum of the PAN/G10 membranes combines the characteristic peaks of the PAN and graphene nanoplatelets (Fig. 2D, black spectrum). In the PAN/G10 membranes, the D, G and 2D bands were shifted upwards to $1353 \mathrm{~cm}^{-1}, 1585 \mathrm{~cm}^{-1}$ and $2707 \mathrm{~cm}^{-1}$, respectively, with respect to the wavelengths of the commercial graphene nanoplatelet spectrum, which is related to the compression of the graphene nanoplatelets caused by the polymer molecules (Yan et al. 2012).

To evaluate the dispersion of graphene nanomaterials on the PAN/G membranes, a specimen of $0.144 \times 0.088 \mathrm{~mm}$ of a PAN/G10 membrane was characterized by Raman spectroscopy. The intensity of the $\mathrm{G}$ band $\left(I_{\mathrm{G}}\right.$, Fig. $\left.2 \mathrm{E}\right)$, the relative intensity of the $\mathrm{D}$ and $\mathrm{G}$ peaks $\left(I_{\mathrm{D}} / I_{\mathrm{G}}\right.$, Fig. $\left.2 \mathrm{~F}\right)$, the ratio of the $2 \mathrm{D}$ and $\mathrm{G}$ peaks $\left(I_{2 \mathrm{D}} / I_{\mathrm{G}}\right.$, Fig. $\left.2 \mathrm{G}\right)$, the ratio of the polymeric $-\mathrm{CN}$ peaks, the graphene content, and the $\mathrm{G}$ peak $\left(I_{\mathrm{CN}} / I_{\mathrm{G}}, \mathrm{Fig} .2 \mathrm{H}\right)$ in the PAN/G10 membrane surface were measured and are depicted in Fig. 2E-H. The homogeneous $I_{2 \mathrm{D}} / I_{\mathrm{G}}$ ratio averaging 0.32 indicated that graphene was present throughout the surface of the membrane and that during sonication, subtle delamination of the original graphene nanoplatelets was produced. Furthermore, an $I_{\mathrm{D}} / I_{\mathrm{G}}$ ratio of 0.25 was found in commercial graphene, and in the PAN/G10 membranes, this ratio was also in the range of 0.15 to 0.5 , indicating that the structural chemistry of the commercial graphene was not altered during membrane processing. However, the one order of magnitude variations in the $I_{\mathrm{CN}} / I_{\mathrm{G}}$ ratios in the range $0.02-0.2$ point to important differences in graphene concentration throughout the membrane surface. The presence of scattered graphene agglomerates $\left(I_{\mathrm{CN}} / I_{\mathrm{G}}\right.$ ratios higher than 0.1 points) can be clearly appreciated on the PAN/G10 membranes (Fig. $2 \mathrm{H}$ and Additional file 2: Fig. S2A "uneven G"). The statistical analysis of the mean Raman $I_{\mathrm{CN}} / I_{\mathrm{G}}$ intensity peak ratio taken over a lattice in the PAN/G10 composites was $0.087 \pm 0.037$ (mean \pm SD). However, when the PAN/ G10 membranes were produced, adapting the conditions to force the homogeneous dispersion of the graphene in the polymer matrix, the $I_{\mathrm{CN}} / I_{\mathrm{G}}$ ratio had a mean value of 
$0.129 \pm 0.026$ (Additional file 2: Fig. S2A, "even G"), which was significantly higher than the "uneven G" $I_{\mathrm{CN}} / I_{\mathrm{G}}$ ratio of the PANG10 membranes $(p=0.000069)$.

\section{Graphene-enriched PAN membranes induce changes in the cell size and shape of MCF7 cancer cells to an epithelial-like phenotype}

Interestingly, we observed an important influence of graphene dispersion on the PAN/ G10 membranes on the cell cultures. Low magnification confocal images (Additional file 2: Fig. S2C, E) showed that cell cultures grown over PAN/G10 with uneven G exhibited a nonhomogeneous cellular distribution over the growth surface, in contrast to "even G" PAN/G10 membranes.

Furthermore, we also investigated the effect of increasing amounts of these graphene agglomerates on the adhesion capacity of tumor cell lines to PAN/G membranes. For this purpose, MCF7 and NSC34 cells were cultured either on glass coverslips (Control) or on "uneven G" PAN/G membranes with $0-10 \%$ w/w graphene (PAN/G0-PAN/G10) (Additional file 3: Fig. S3A-D, F-I, respectively). We determined that, in contrast to MCF7 cells, the presence of $10 \%$ graphene was required for the adhesion of NSC34 to PAN/G membranes and, therefore, established that these membranes (hereafter PAN/G10) provided optimal adhesion properties for cell lines with minimal cytotoxicity (Additional file 3: Fig. S3E, J). Because they also present optimum electrical properties, we selected PAN/G10 membranes for our experimental studies.

To determine the presence of possible changes in cell size and shape induced by PAN/G10, we stained the cultured cells with phalloidin-FITC, a cytochemical marker of polymerized actin microfilaments. Whereas control MCF7 and NSC34 cells exhibited a star shape with several cellular processes, the cells on the PAN/G10 substrate lost their extensions and became polygonal, consistent with an epithelial-like phenotype, and formed cell clusters (Fig. 3A-D). Planimetric determination of the cell surface area showed a PAN/G10-dependent significant reduction in cell size (Fig. 3E). Similarly, the estimation of the circularity shape factor $4 \pi A / P^{2}$ ( $A$ is the surface area and $P$ the perimeter), which is unity for a rounded cell shape and close to zero for a flattened fusiform cell shape, revealed a significant increase in this factor in cells cultured on PAN/G10 (Fig. 3F). This finding reflects the acquisition of a more regular, epithelial-like, polygonal cell shape, which requires a spatial reorganization of cytoskeleton beneath the plasma membrane and de novo formation of intercellular junctions.

These observations were also confirmed in other cancer cell types derived from neuroblastoma (SH-SY5Y, Additional file 4: Fig. S4A, B) and glioblastoma (U87 and C6, Additional file 4: Fig. S4C-F). HeLa cancer cervix cells also showed a dramatic decrease in cell size and a more rounded cell shape but without cell cluster formation (Additional file 4: Fig. S4G, H). In contrast, nontumor-derived cells, such as immortalized mouse embryonic fibroblasts (MEFs, Additional file 4: Fig. S4I, J) or human normal mesenchymal cells (ASC52, Additional file 4: Fig. S4K, L), did not exhibit major alterations in cell shape or in their F-actin stress fiber cytoskeleton when grown on PAN/G10 membranes.

Next, we investigated whether the presence of PAN/G10 induced cytotoxicity with a TUNEL assay for apoptosis. As shown in Fig. 3G, a minimal proportion of both MCF7 and NSC34 cells, approximately $1-3 \%$, cultured either in control medium or on a PAN/ 

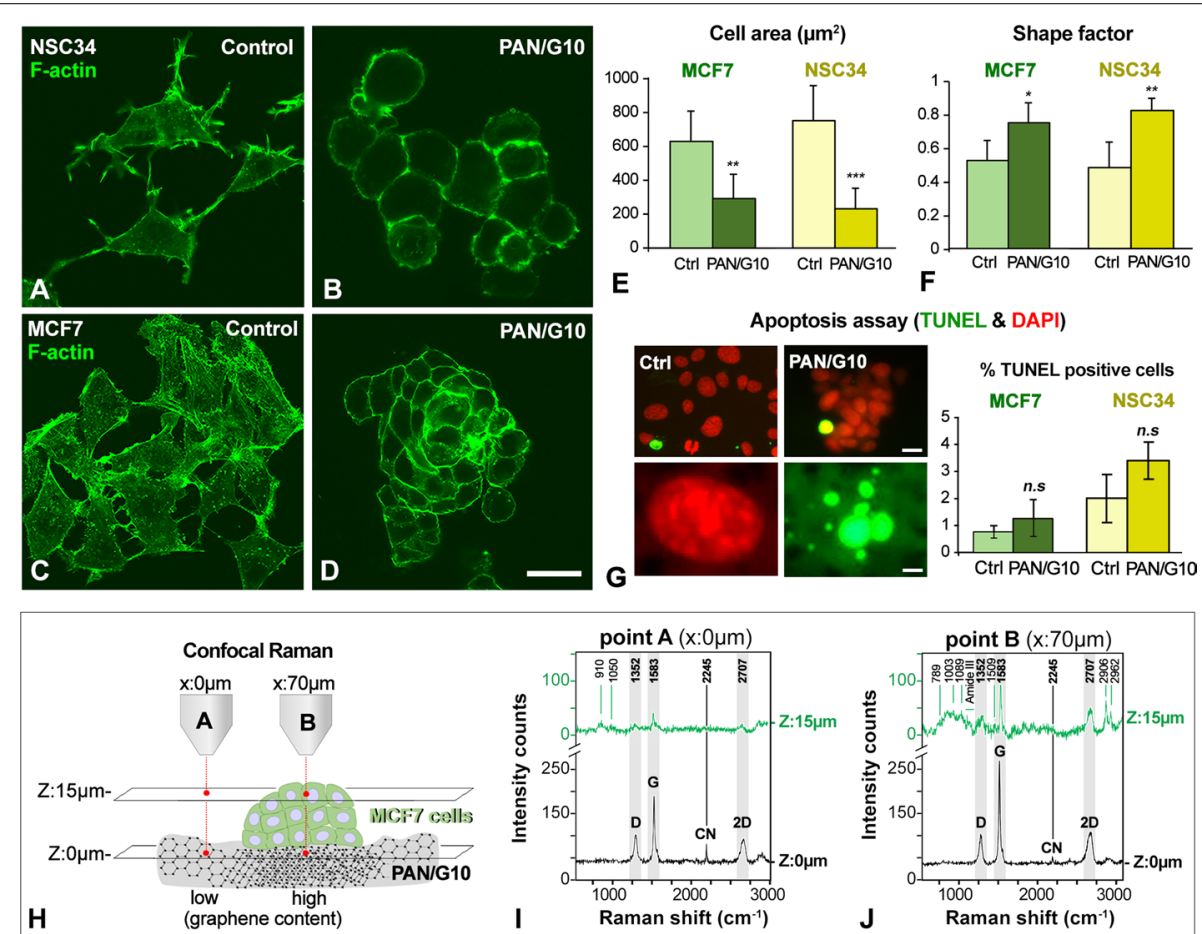

Fig. 3 A-D Confocal microscopy images of NSC34 (A, B) and MCF7 (C, D) cells cultured on glass coverslips or on PAN/G10 membranes and counterstained with FITC-conjugated Phalloidin to reveal F-actin distribution. E, F Morphometric analysis of MCF7 and NSC34 cells 2 days after culture on glass coverslips (Ctrl) or on PAN/ G10 membranes. Fluorescence images were taken from cells counterstained with Phalloidin-FITC and the surface area $(\mathbf{E})$ and the shape factor $(\mathbf{F})$ were measured from at least 50 cells of each experimental group. Bars represent means \pm SD. G Apoptosis assay was performed by using TUNEL staining of MCF7 and NSC34 cells grown for 2 days on glass coverslips (Ctrl) or on PAN/G10 membranes. Cells were counterstained with DAPI (red signal) and the percentage of TUNEL-positive cells (green signal) was calculated. A detail of a normal nucleus and a typical apoptotic TUNEL-positive cell are shown. Bars represent mean \pm SD. $\mathbf{H}$ Schematic depiction of the points $A$ and $B$ (separated by $70 \mu \mathrm{m}$ in distance over the membrane surface) and altitudes (with the laser focusing at $Z=0 \mu \mathrm{m}$ meaning at the level of the membrane surface and at $Z=15 \mu \mathrm{m}$ over the membrane surface level) where Raman spectra were acquired for a PAN/G10 membrane with MCF7 clusters formed on top after 2 days of cell culture. I, J Raman spectra of the point A and B, respectively. I Point A spectrum shows a region of the membrane surface $(Z=0 \mu \mathrm{m})$ with low concentration of graphene ( $G$ band) relative to polymer ( $C N$ peak intensity). Characteristic bands representative of biomolecules present on cells are not detected at $Z=15 \mu \mathrm{m}$ in point $A$. $\mathbf{J}$ Point $B$ spectra at $Z=0 \mu \mathrm{m}$ is representative of a region with high agglomeration of graphene (low $I_{C N} / I_{G}$ ratios) where, otherwise, at $Z=15 \mu \mathrm{m}$ typical bands of biomolecules in cells were clearly appreciated. ${ }^{*} p<0.05,{ }^{* *} p<0.005,{ }^{* * *} p<0.0005$, n.s: not significant. Scale bars: $10 \mu \mathrm{m}(\mathbf{A}-\mathbf{D}$ and $\mathbf{G})$

G10 membrane underwent apoptosis, and the difference between the control and PAN/ G10 cultures was not significant.

\section{Cells preferentially localize on graphene-rich regions of PAN/G10 membranes}

To investigate whether the MCF7 cancer cell clusters localized preferentially on graphene-rich regions or PAN polymer-rich regions, the Raman spectra of two different locations (points $A$ and $B$ ) and at two different altitudes $(Z=0$ and $15 \mu \mathrm{m})$ over the membrane surface were taken as schematically depicted in Fig. $3 \mathrm{H}$. The analysis of the spectrum of point $\mathrm{A}$ at the $Z=15 \mu \mathrm{m}$ location in the membrane revealed that cells were not present at this point. The peaks at 910 and $1050 \mathrm{~cm}^{-1}$ were previously attributed to PBS 
(Gullekson et al. 2011). Signals corresponding to the D, G and 2D bands (1352, 1583 and $2707 \mathrm{~cm}^{-1}$, respectively) from the resonance of the PAN/G10 membrane were equally observed (Fig. 3I). On the other hand, the Raman spectrum corresponding to point B at $Z=15 \mu \mathrm{m}$ over the membrane surface showed important signals of characteristic bands of biomolecules, confirming the presence of MCF7 cells (Fig. 3J). The bands at 2906 and $2962 \mathrm{~cm}^{-1}$ were assigned to $\mathrm{CH}_{2}$ vibrations of lipids and $\mathrm{CH}_{3}$ vibrations of proteins (Abramczyk et al. 2015; De Vitis et al. 2016). Peaks at 789, 1089 and $1509 \mathrm{~cm}^{-1}$ can be ascribed to ring breathing of cytosine, $\mathrm{PO}_{2}^{-}$symmetric stretching in nucleic acids, and the band vibration of the imidazole ring relative to the nucleic acid bases, respectively (Draux et al. 2010; De Vitis et al. 2016). Additionally, other protein-related spectral features were also present, such as the phenylalanine ring breathing mode $\left(1003 \mathrm{~cm}^{-1}\right)$ and the amide III spectral feature (between 1250 and $1325 \mathrm{~cm}^{-1}$ ). The appearance of D and G bands characteristic of graphene in the PAN/G10 membranes might be superimposed on other important bands characteristic of biomolecules, hampering their identification. Interestingly, the $I_{\mathrm{CN}} / I_{\mathrm{G}}$ ratios calculated from the spectra of points $\mathrm{A}$ and $\mathrm{B}$ at $Z=0 \mu \mathrm{m}$ were 0.22 and 0.05 , respectively. This indicated that cell clusters localized preferentially on points of the membrane surface with a high concentration of graphene.

As previously indicated, PAN/G10 membranes with uneven and even G distributions were prepared in the present study (Additional file 2: Fig. S2B, D). A comparison between the morphology of MCF7 clusters in these different PAN/G10 membranes is shown in Additional file 2: Fig. S2C, E. PAN/G10 membranes with an uneven G distribution completely avoided the formation of actin-based membrane protrusions of the cell edge, such as filopodia and lamellipodia, that control cell movement; therefore, defined rounded cell clusters were clearly observed on these types of PAN/G10 membranes (Additional file 2: Fig. S2C).

PAN/G10 promotes the transition of MCF7 cells to an epithelial-like cell phenotype induced by the upregulation of key epithelial molecular markers and the formation of adherens junctions

Next, we further investigated the effect of PAN/G10 on the association behavior of MCF7 cells using fluorescence microscopy analysis. Whereas control MCF7 cells exhibited an irregular morphology and did not establish adherens junctions (Fig. 4A, G), cells cultured on PAN/G10 membranes progressively aggregated into cellular islets (Fig. 4B) that resembled multicellular spheroid-like structures when observed using three-dimensional reconstruction of confocal images (see Additional file 6: Video S1). Confocal and live-cell microscopy revealed that the volume of these cellular islets increased over time due to cell proliferation and recruitment of neighboring cells (see Fig. 4B-D and Additional file 7: Video S2 and Additional file 8: Video S3). Notably, these MCF7 islets were solid structures (without a lumen), as observed by confocal serial image acquisition of the whole islet 3D volume (Additional file 5: Fig. S5).

To further understand these graphene-induced phenotypic changes, we analyzed the expression of the $I D 1$ gene, which encodes a transcriptional regulator involved in MET (Stankic et al. 2013). RT-qPCR determination of ID1 mRNA levels revealed a dramatic increase, by approximately 14 times, in MCF7 cellular islets formed on the PAN/ G10 membranes relative to the control cells (Fig. 4E). Similarly, mRNA expression of 

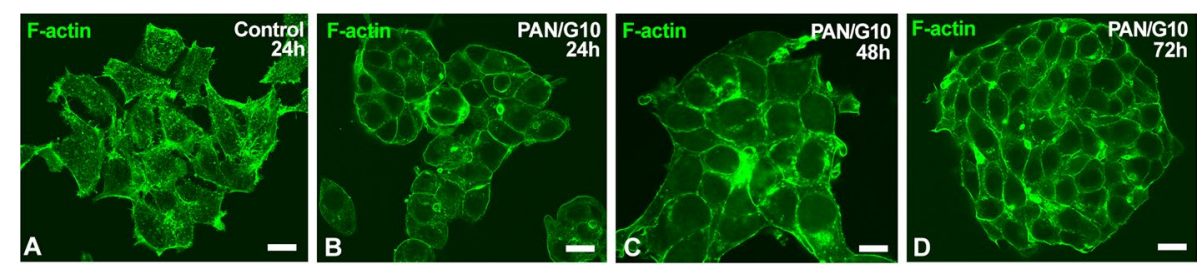

ID1 KRT18

TJP1

EPCAM ACTB

CTNNB1
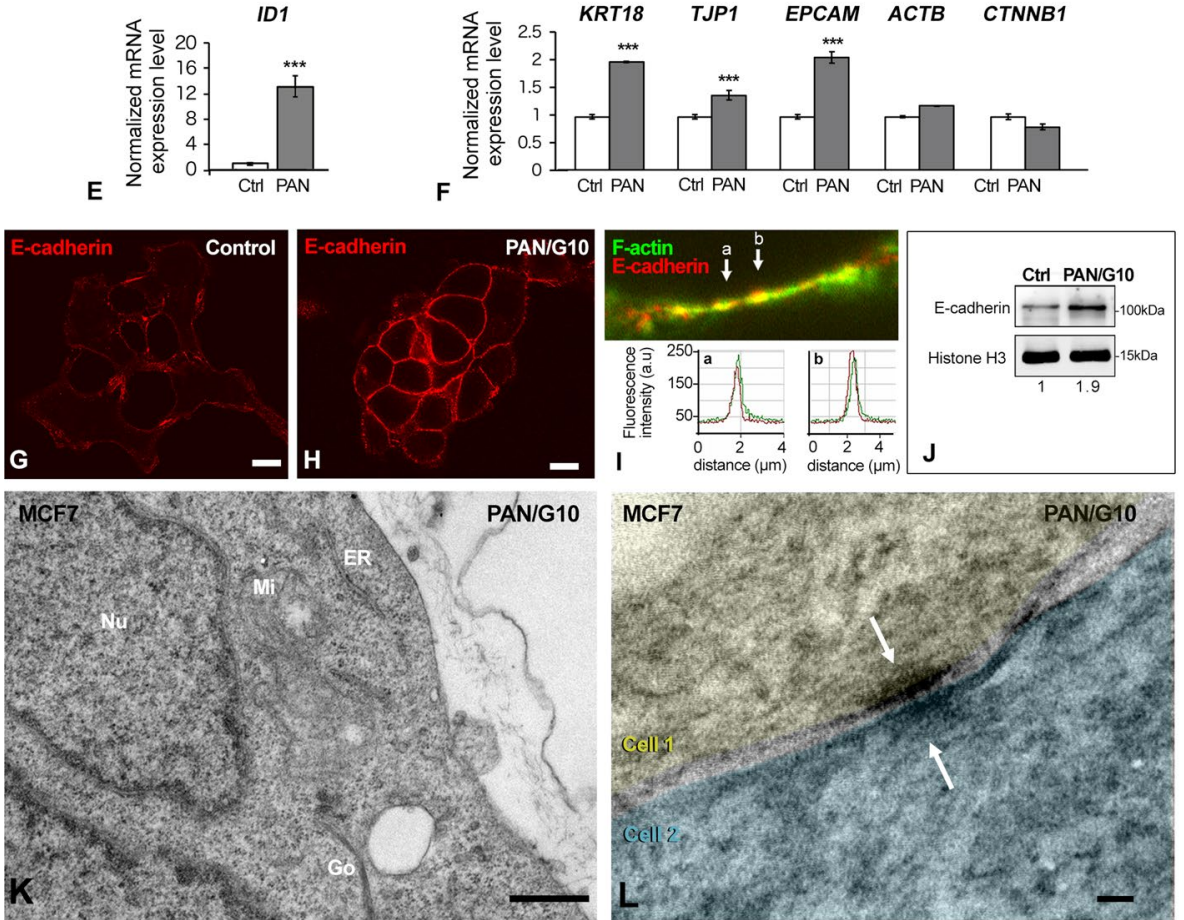

Fig. 4 A-D Confocal images showing the actin filaments (F-actin) of the cytoskeleton of MCF7 cells grown on glass coverslips (Control, A) or PAN/G10 membranes for 24 (B), 48 h (C) or 72 h (D). Note the cellular aggregation induced by PAN/G10 forming cellular islets that grow significantly in size over time (72 h). E, F RT-qPCR determination of ID1 (E), KRT18, TJP1, EPCAM, CTNNB1, ACTB (F) gene expression in MCF7 cells RNA extracts grown on glass coverslips (Ctrl) or on PAN/G10 membranes as indicated. Bars represent means \pm SD of three independent experiments. G, $\mathbf{H}$ Immunofluorescence and confocal microscopy images showing E-cadherin expression in cell membrane of MCF7 breast cancer cells grown on control coverslips or on PAN/ G10 membranes. Note how the increased signal of E-cadherin at the edges of MCF7 cells forming islets due the PAN/G10 induced formation of adherens junctions. I The confocal image shows a detail of the cellular edge from two cells co-stained for E-cadherin (red signal) and Phalloidin-FITC (green signal), to reveal F-actin. The plots represent the linear profile of fluorescence intensity signals of E-cadherin (red) and F-actin (green) at the indicated points $(a, b)$ in the image. Note the co-localization and similar intensity of both signals indicating the presence of intercellular E-cadherin-positive adherens junctions that normally are enriched in actin filaments (F-actin). J Western blotting analysis showing upregulation of E-cadherin expression in MCF7 cells grown on normal Petri dishes (Ctrl) or on PAN/G10 membranes. Histone H3 was used as loading control. The relative fold-increase of E-cadherin was calculated using Image J software. $\mathbf{K}$, $\mathbf{L}$ Transmission electron micrographs of MCF7 cells grown on PAN/G10 membranes. Note, in panel (K), the normal appearance of the typical cellular organelles such as mitochondria (Mi), endoplasmic reticulum (ER), Golgi apparatus (Go), as well as the lack of heterochromatin clumps in the nucleus (Nu), of the epithelial-like cells which form cellular islets. The higher magnification detail in (L), shows a typical intercellular adherens junction (arrows). ${ }^{* * *} p<0.0005,{ }^{* *} p<0.005,{ }^{*} p<0.05, n . s$ not significant. Scale bar: $15 \mu \mathrm{m}(\mathbf{A}-\mathbf{D}$ and $\mathbf{G}, \mathbf{H}), 3 \mu \mathrm{m}(\mathbf{K})$ and $500 \mathrm{~nm}$ (L)

three key epithelial markers, ZO-1 (tight junction protein), cytokeratin 18 (epithelial intermediate filaments) and EpCAM (epithelial cell adhesion molecule), were upregulated in MCF7 cells cultured on the PAN/G10 membranes (Fig. 4F). Furthermore, the 
expression of genes encoding $\beta$-actin $(A C T B)$, main component of the actin cytoskeleton, and $\beta$-catenin (CTNNB1), a bifunctional protein that acts as a transcription factor or intercellular adhesion molecule, was not significantly changed in MCF7 cells cultured on PAN/G10 membranes relative to control cells.

Altogether our results suggest that growth of MCF7 breast cancer cells on graphenerich regions induces a transition to an epithelial-like phenotype. This was further confirmed by the observation that MCF7 cells within the islet appeared closely packed by band-type adherens junctions immunolabeled for E-cadherin that completely encircled the cells (Fig. 4G, H). Moreover, the adherens junctions were closely associated with the phalloidin-FITC-stained actin filaments that also surrounded the cells just below the plasma membrane (Fig. 4I). Linear profiles of fluorescence intensity signals of actin and E-cadherin along the adherens junctions confirmed the co-localization of both molecules (Fig. 4I). The increased expression of E-cadherin in MCF7 cells cultured on PAN/ G10 was validated by western blotting of the cell lysates (Fig. 4J). Furthermore, electron microscopy examination showed flattened cells with euchromatic nuclei, normal cytoplasmic organelles and the presence of adherens junctions (Fig. 4K, L).

Collectively, our findings on the association of MCF7 cells in islets, together with the increased expression of E-cadherin protein and ID1, TJP1 (ZO-1), EPCAM (EpCAM) and KRT18 (cytokeratin 18) mRNAs, suggest that graphene-rich biomaterials induce a transition of MCF7 breast cancer cells from a mesenchymal to an epithelial-like phenotype.

Downregulation or inactivation of $C D H 1$ gene in solid tumor cells, including breast cancer, is thought to be involved in their increased cell migration and invasion capacity during metastasis (Baranwal and Alahari 2009; Moll et al. 1993; Tycko 2000; Vesuna et al. 2008; Zhang et al. 2013; Onder et al. 2008; Stankic et al. 2013; Andrews et al. 2012; Canel et al. 2013). Therefore, we next investigated whether MCF7 cells cultured on PAN/G10 membranes upregulated $C D H 1$ expression. RT-qPCR determination of $C D H 1$ mRNA levels revealed a significant increase in MCF7 cells cultured on PAN/G10 membranes relative to control cells (Fig. 5A). This finding supports the hypothesis that the presence of graphene promotes transcriptional activation of the E-cadherin-encoding gene.

Interestingly, in addition to transcriptional regulation, downregulation of E-cadherin expression can be produced by epigenetic modification. Specifically, the CpG island of $C D H 1$ promoter has been reported to be highly methylated in a variety of human cancers and mesenchymal cell lines, including breast cancer (Corso et al. 2020; Ding et al. 2012; Droufakou et al. 2001; Nass et al. 2000). In this line, we examined whether the upregulation of the CDH1 gene in MCF7 cells grown on PAN/G10 was associated with changes in the methylation status of selected regions from the promoter of this gene. The $C D H 1$ gene contains one large (1735 bp) CpG island that covers the region from -406 to +1329 regarding the position of the transcription starting site (TSS) (Fig. 5B). The CpG dinucleotides selected for DNA methylation analysis and its genomic position, as well as the primers used in this study, are shown in Fig. 5B (inset). Methylation sensitive PCR (MSP) analysis showed that $100 \%$ of gDNA from MCF7 control cells was methylated in the CpG positions located within the primer positions $(-154$ and -152$)$. However, when MCF7 were grown for $48 \mathrm{~h}$ in PAN/G10 membranes the MSP analysis showed the presence of a small fraction (4\%) of unmethylated DNA in the analyzed 


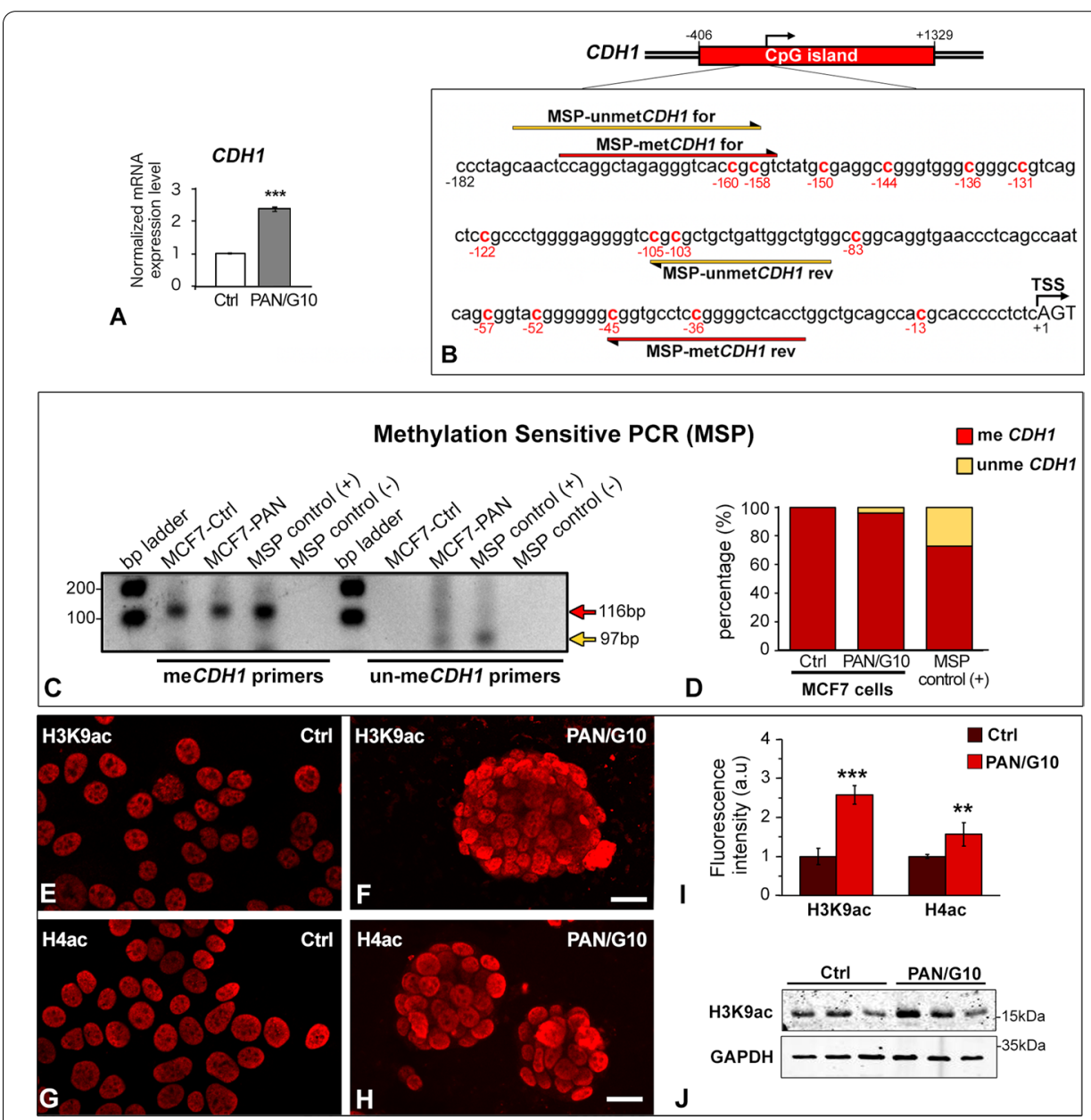

Fig. 5 A mRNA expression levels of $C D H 1$ in MCF7 cells $(n=3)$ grown on control culture plates (Ctrl) or on PAN/G10 membranes (PAN) was determined by qRT-PCR. mRNA levels are relative to that of GAPDH and are expressed as mean \pm SD. $\mathbf{B}$ Schematic representation of part of the E-cadherin gene $\mathrm{CDH} 1$ encompassing a CpG island (from -406 to + 1329). Inset shows the detailed sequence of the region analyzed by Methylation Sensitive PCR (MSP). The position of the Transcription Starting Site (TSS) is marked as " +1 ", the position of cytosines in $\mathrm{CpG}$ dinucleotide are colored in red and their position is indicated. The positions of primers used for analysis of methylated or unmethylated DNA by MSP are shown in the inset. C MSP was performed using bisulfite-converted genomic DNA (gDNA) from MCF7 cells grown on control culture plates (MCF7-Ctrl) or on PAN/G10 membranes (MCF7-PAN). As positive control (MSP-control (+) we used bisulfite-converted gDNA from normal human tissue samples and as negative control (MSP-control (-)), gDNA was excluded from the $P C R$ reaction. The PCR products obtained after PCR amplification using the two primer sets designed for recognition of methylated (116 bp) or unmethylated DNA ( $96 \mathrm{bp}$ ) were run in a $2 \%$ agarose gel and the image was acquired using a GelDoc system (Biorad). D 100\% Stacked bars graph depicting the percentage of methylated (red) and unmethylated (yellow) DNA. Band quantification was performed after background removal using ImageJ software. $\mathbf{E}-\mathbf{H}$ Immunofluorescence and confocal microscopy images showing H3K9ac $(\mathbf{E}, \mathbf{F})$ and $\mathrm{H} 4 \mathrm{ac}(\mathbf{G}, \mathbf{H})$ expression in cell membrane of MCF7 breast cancer cells grown on control coverslips or on PAN/G10 membranes. I Quantitative measurement of mean fluorescence intensity per nuclear area after background removal. Data were obtained from three different images with more than 100 cells per image using Image $(\mathrm{NIH})$ software. Bars represent mean \pm SD. J Western blotting analysis showing H3K9ac, expression in MCF7 cells grown on normal Petri dishes $(n=3$, Ctrl) or on PAN/G10 membranes $(n=3$, PAN/ G10). GAPDH was used as loading control

CpG positions (Fig. 5C, D). This finding support that a slight but nonsignificant demethylation occurs at the selected $C D H 1$ promoter region in MCF7 cultured on PAN/G10 membranes. 
In addition to DNA methylation patterns, epigenetic changes in nucleosomal histone acetylation profiles have been involved in breast cancer (Guo et al. 2018; Jawaid et al. 2010; Lustberg and Ramaswamy 2011; Sadikovic et al. 2008; Zhuang et al. 2020). On this basis, we investigated whether the transition of MCF7 cells cultured on PAN/G10 membranes to an epithelial-like phenotype was associated with global changes in acetylation status of nucleosomal histones $\mathrm{H} 3$ and $\mathrm{H} 4$. The immunofluorescence analysis revealed that MCF7 cells grown on graphene-rich regions showed increased expression of both acetylated histones, $\mathrm{H} 3$ on lysine 9 (H3K9ac) and pan-acetylated histone $\mathrm{H} 4$ on lysines 5, 8, 12 and 16 (H4ac), compared with control cells (Fig. 5E-H). This finding was confirmed by densitometric analysis of nuclear fluorescence signal intensity of H3K9ac and H4ac (Fig. 5I) and western blotting of H3K9ac protein levels (Fig. 5J). Enhanced histone acetylation has long been linked with an open chromatin structure and transcriptionally active genes (Clayton et al. 2006).

\section{PAN/G10 induces a migrastatic response in MCF7 breast cancer cells}

Cell migration plays a key role in the steps of invasion and metastasis of cancer cells. The migratory activity of these cells in solid cancer depends on the intrinsic properties of tumor cells. They include deregulation of the actin cytoskeleton, loss of E-cadherin expression and altered expression of integrins and metalloproteases, as well as changes in cell-extracellular matrix interactions (Hanahan and Weinberg 2011; Weinberg 2014). To determine the effects of graphene on the migratory behavior of MCF7 cells, we used a two-dimensional wound healing assay (Pijuan et al. 2019) in the absence (Fig. 6A, B) or presence of PAN/G10 (Fig. 6C, D). Images of the wounded areas at 0 and $24 \mathrm{~h}$ are

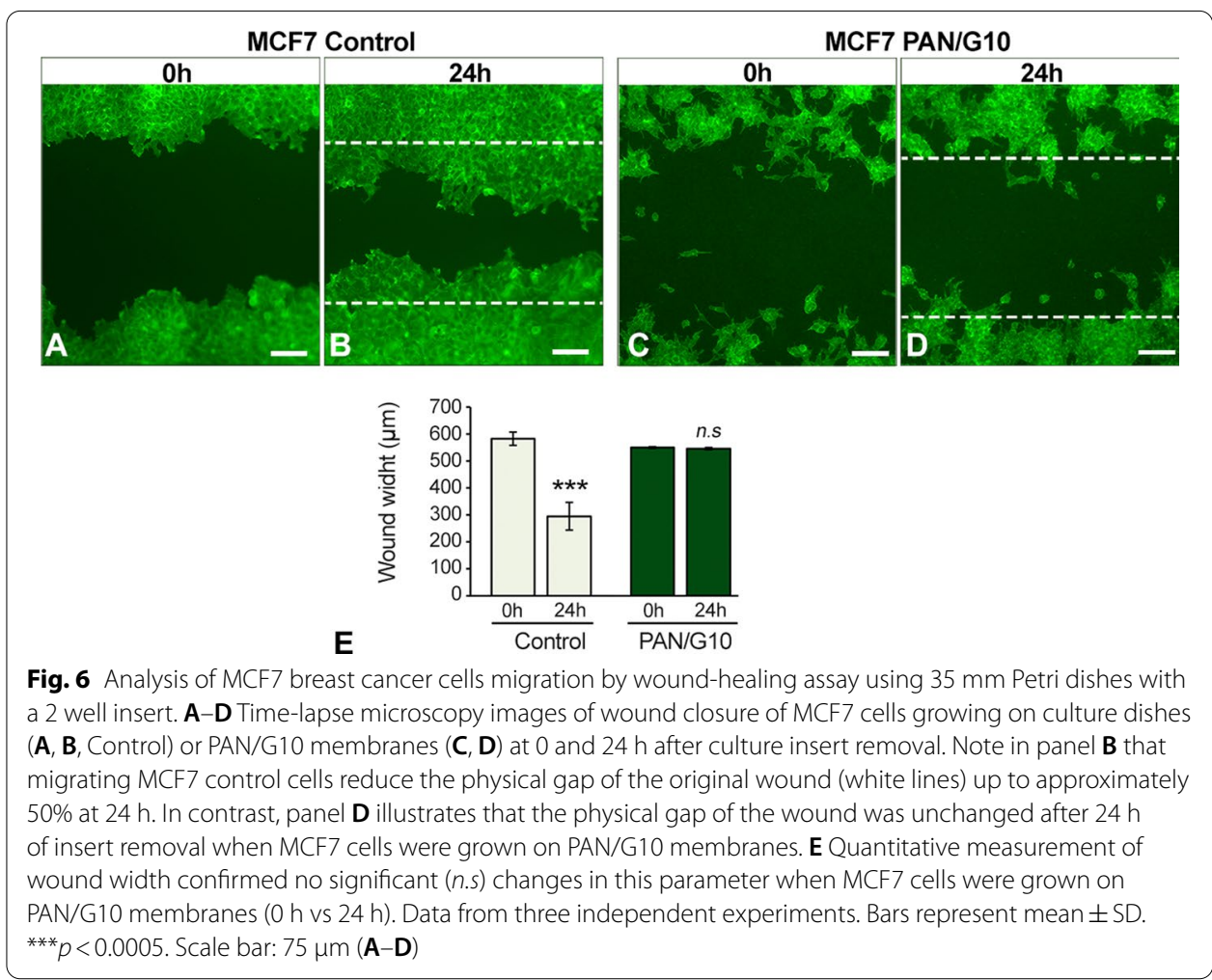


illustrated in Fig. 6. Measurements of the wound width showed that the scratch was half closed within $24 \mathrm{~h}$ in control cultures, whereas nonsignificant migratory activity was detected in MCF7 cells cultured on PAN/G10 membranes (Fig. 6E). In conclusion, the biophysical properties of graphene induce a rearrangement of cells to an epithelial-like transition with increased cell cohesion and inhibition of migratory activity in the human breast cancer MCF7 cell line.

\section{Discussion}

It is well established that cancer cells, through the progression of several genetic and epigenetic events, acquire several cellular hallmarks shared by most human tumors. Among others, they include abnormal growth, resistance to cell death, genome instability and the ability to invade and metastasize different organs (Hanahan and Weinberg 2011; Weinberg 2014). Our study indicates that MCF7 human breast carcinoma cells cultured on PAN/G10 membranes differentiate into an epithelial-like phenotype through three essential mechanisms: (i) an increase in cell adhesion, mediated by the upregulation of E-cadherin, ZO-1, EpCAM and cytokeratin 18 encoding genes; (ii) activation of ID1 gene expression involved in MET (Stankic et al. 2013), and (iii) inhibition of the migratory ability of the cells. The adhesion and migrastatic (Gandalovičová et al. 2017) effects of graphene on these tumor cells, support the therapeutic potential of graphene and graphene derivatives for effective treatments of certain human tumors.

Using confocal Raman spectroscopy, an emerging nondestructive and label-free technique to study the biochemical and biophysical properties of cells (Manciu et al. 2017), we found that the Raman spectrum of the PAN/G10 membranes was unevenly distributed throughout the membrane surface. We took advantage of this fact to demonstrate that MCF7 cell islets were preferentially localized on graphene-rich regions of the PAN/ G10 membranes. This observation suggests that the physicochemical, particularly electrical, properties of graphene are presumably able to trap tumor cells into cell clusters or islets, resulting in increased cellular adhesion and inhibition of cell motility.

Regarding the biophysical and biochemical properties of graphene, all PAN/G substrates presented water contact angles below $60^{\circ}$ (maximum in PAN/G0 membranes with $56^{\circ} \pm 4^{\circ}$ and $53^{\circ} \pm 5^{\circ}$ for PAN/G10 membranes), which indicated the hydrophilicity of these materials due the incorporation of hydrophilic graphene at high concentrations. Graphene materials with water contact angles of $64^{\circ}$ were previously reported to enhance $\mathrm{C} 2 \mathrm{C} 12$ cells adhesion, as it potentially favors serum protein adsorption (Bajaj et al. 2014). Furthermore, the remarkable protein loading capacity of graphene and graphene derivative nanomaterials is well known to be due to their establishment of $\pi-\pi$ stacking noncovalent interactions (Chen et al. 2018). Graphene might bind to globular extracellular proteins and glycoproteins (albumin and fibronectin) present in serum, which are key intermediate molecules for cell adhesion on polymeric substrates that ultimately enhance cell adhesion as the content of graphene increases on PAN/G membranes.

Importantly, the high concentration of graphene in the PAN/G10 membranes did not induce apoptosis-mediated cytotoxicity in MCF7 or NSC34 cells. Furthermore, the adhesion capacity of NSC34 was increased on PAN/G10 compared to graphene-free PAN membranes, in agreement with previous works that reported improved human 
mesenchymal stem cell (hMSC) adhesion on chitosan-reduced graphene oxide (rGO) composite films with increasing rGO concentrations from 0 to 5\% (Kim et al. 2013).

Our results indicate that the upregulation of E-cadherin expression is a major factor in the adhesive phenotype of MCF7 cells cultured on PAN/G10 membranes. E-cadherin is a calcium-dependent transmembrane linker protein that plays a crucial role in the maintenance of the tissue architecture, particularly of the adhesive and polarized properties of epithelial cells (Gumbiner 1996; Pálmer et al. 2001; Ramirez Moreno et al. 2021). In addition to forming homotypic cell-cell interactions, E-cadherin may contribute to regulating cell growth and differentiation by interacting with multifunctional $\beta$-catenin (Tycko 2000). E-cadherin is considered a tumor suppressor, and its loss promotes tumor metastatic dissemination and predicts poor prognosis (Gulliford et al. 1998; Hanahan and Weinberg 2011; Pálmer et al. 2001; Venza et al. 2016).

In cancer pathogenesis, E-cadherin gene may be silenced epigenetically via DNA methylation of cytosine in repetitive promotor-associated CpG islands (Corso et al. 2020; Droufakou et al. 2001; Tycko 2000). For instance, hypermethylation of CpG islands in promoter of $C D H 1$ gene, associated with a decrease in E-cadherin expression, frequently occurs in human breast cancer, as well as in certain breast cancer cell lines (Caldeira et al. 2006; Nass et al. 2000). Our study showed nonsignificant changes in the methylation status of $\mathrm{CDH1}$ gene at the promoter $\mathrm{CpG}$ dinucleotides analyzed in MCF7 cells cultured on PAN/G10. However, we cannot rule out that epigenetic variations of $C D H 1$ gene may occur in other $\mathrm{CpG}$ sites of this gene.

The upregulation of genes encoding key epithelial markers (E-cadherin, ZO-1, EpCAM, cytokeratin 18) in MCF7 cells cultured on graphene-rich biomaterials is consistent with the increased levels of acetylated histones $\mathrm{H} 3$ and $\mathrm{H} 4$ found in the present study. In fact, post-translational modifications by acetylation of histones $\mathrm{H} 3$ and $\mathrm{H} 4$ are usually linked with an open chromatin structure and active genes (Clayton et al. 2006). In this context, our results suggest that E-cadherin expression could be modulated by signaling pathways activated by the presence of graphene in the extracellular microenvironment. We propose that these signaling pathways may upregulate the expression of genes encoding epithelial cell markers involved in the transition to an epithelial-like phenotype.

Also noteworthy is the absence of significant changes in the expression of genes encoding $\beta$-actin and $\beta$-catenin in MCF7 cells cultured on PAN/G10 relative to control cells. Sustained expression of these genes may be necessary for the MET. This cellular event should require a partial redistribution of $\beta$-actin and $\beta$-catenin to plasma membrane for cell adhesion (Green et al. 2010) rather than a change in the transcriptional activity of their encoding genes. In this sense, the transition to a polygonal shape found in MCF7 cells cultured on PAN/G10 may be related with the strength of intercellular cohesion in MCF7 cell islets. In fact, the intercellular cohesion and adhesion in epithelial-like MCF7 cells should require a partial reorganization of cytoskeletal architecture beneath the plasma membrane and de novo formation of intercellular junctions which can influence the cell shape.

Previously, MCF7 cells cultured in a scaffold-free system that self-assembled into 3D microtissues were found to have different gene expression, adhesion and differentiation patterns compared to those cultured on 2D monolayers (Vantangoli et al. 2015). Thus, 
MCF7 microtissues have a luminal space, similar to acini of the breast glands, exhibit increased expression of some epithelial markers, form adherens junctions and maintain their estrogen responsiveness (Vantangoli et al. 2015). Although the MCF7 islets on PAN/G10 membranes presented similar morphology and adhesive properties to those of 3D microtissues (Vantangoli et al. 2015), luminal formation was not observed.

Previous works have demonstrated that micro- and nanotopography of artificial celladhesive poly(dimethyl siloxane) (PDMS) substrates affected MET through epigenetic reprogramming (Downing et al. 2013). The biomechanical cues induced by PDMS substrates with microgrooves of $10 \mu \mathrm{m}$ width and $10 \mu \mathrm{m}$ spacing significantly improved the efficiency of the early-stage cell reprogramming of mouse fibroblasts toward iPSCs. However, to achieve complete iPSC reprogramming, additional chemical or reprogramming factors were deemed necessary. Improved pluripotent reprogramming efficiency was also observed with Oct4-GFP knock-in (KI) mouse embryonic fibroblasts (MEFs) cultured on graphene-coated substrates generated by chemical vapor deposition (Yoo et al. 2014). Overall, the evidence collected proved that graphene substrates can induce efficient cell reprogramming via the activation of the mesenchymal-to-epithelial transition. The mechanisms behind the relationship between graphene and epigenetic changes are still unclear.

In the present study, PAN/G10 substrates also seemed to activate MET in MCF7 cells, as indicated by the dramatic upregulation of the ID1 gene, which encodes a transcriptional regulator involved in cell differentiation via MET (Stankic et al. 2013). A major breakthrough is that PAN/G10 membranes spontaneously induce an epithelial-like transition upon cell-substrate contact in the absence of additional transcription factors or molecules, and this effect is reproducible in different cancer cell lines.

The preferential localization of MCF7 clusters or islets on graphene-rich regions highlights the impact of graphene on cell fate and differentiation. Actin is an electrically conductive protein (Gharooni et al. 2019). PAN/G10 membranes present higher conductivity than PAN/G0 membranes due to the graphene loading. Graphene-rich aggregates with high adsorption capacity for binding proteins present in serum would favor the adhesion of MCF7 breast cancer cells. The electrically active graphene-rich domains are surrounded by insulating polymer areas impeding the protrusions of conductive actin protein outside the graphene domains. Therefore, graphene islets would be acting as electrical traps of the cancer cells. Regarding the features incorporated by graphene into the PAN/G10 membranes that might trigger the observed cell responses, it is interesting to highlight that the graphene used in the present study is exfoliated graphite with 5-10 graphene layers, according to the supplier's specifications. The exfoliation and other characteristic Raman features of graphene, e.g., low $I_{\mathrm{D}} / I_{\mathrm{G}}$ ratio in the range of 0.15-0.43, were maintained after loading on the PAN/G10 membranes. Interestingly, this graphene is an affordable nanomaterial that can be produced at a large scale through mechanical exfoliation.

The preferential binding interactions of graphene and graphene oxide with differentiation factors, such as dexamethasone or insulin, have been claimed to be the main mechanism favoring osteogenic or adipogenic differentiation, respectively, over PDMS films (Downing et al. 2013). However, the results presented for PAN/G10 membranes were obtained in the absence of chemical differentiation factors in the culture medium. 
Remarkably, the SEM images revealed irregular and rough characteristics of the PAN/ G10 membranes at the microscale in contrast to the PAN/G0 membrane surface topography. It has been previously demonstrated that the nanotopography of extracellular matrix (ECM) or artificial ECM substrates has important effects on cell-substrate interactions. For example, cell-substrate interactions trigger the mechanisms that control the formation and maturation of focal adhesions (FAs) and the subsequent signaling process that promotes neuronal differentiation of $\mathrm{PC} 12$ cells growing on nanogratings (Ferrari et al. 2011). Moreover, rGO-chitosan composite films with 5\% rGO showed improved osteogenic differentiation of hMSCs in comparison to plain chitosan films, which is due to the cues of nanoscale stiffness and roughness attained by the incorporation of graphene into the polymer matrix (Kim et al. 2013). In this context, the cell morphology observed in mesenchymal cells cultured on PAN/G10 membranes was similar to that reported by Kim et al. (2013). These authors observed overexpression of matrix metalloproteinase (MMP)-cleaved osteopontin (OPN) in hMSCs on rGO-chitosan in comparison to cells cultured on plain chitosan substrates, both with and without osteogenic differentiation media. Similarly, micro- and nanotopography of the substrates are considered to be ideal for supporting somatic cell reprogramming on polymer (Downing et al. 2013) or CVD graphene platforms (Yoo et al. 2014).

Notably, the inhibition of migratory activity in human breast cancer MCF7 cells cultured on PAN/G10 supports a "migrastatic" effect (Gandalovičová et al. 2017) of graphene. Previous studies in solid cancer have shown that the migratory activity of these cells depends on the intrinsic properties of tumor cells, including the ROCK pathwaydependent dysregulation of actin dynamics, which influences actin polymerization and actomyosin contractility, loss of E-cadherin, altered expression of integrins and metalloproteases and changes in cell-extracellular matrix interactions (Weinberg 2014; Kümper and Marshall 2011; De Craene and Berx 2013; Masi et al. 2020; Sanz-Moreno et al. 2011).

In this context, the effect of the shape of graphene oxide (GO) micropatterns in glass substrates on cell migration has been previously reported (Kim et al. 2016). Thus, the triangular geometries of micropatterned GO substrates enhanced the cell migration speed, distance and directionality compared to square micropatterns. Cell lamellipodia tended to reach sharp edges and then jump to the closest GO micropattern separated by a distance of $17 \mu \mathrm{m}$ (Kim et al. 2016). These findings suggested that cell migration could be regulated with geometrical cues without chemical factors. Thus, the graphene-rich regions on the PAN/G10 membranes reported here could act similarly to graphene islets or round micropatterns. As the cells could not find any sharp edges here, their migration speed and directionality could be restricted even more than in square micropatterns. This interpretation is consistent with the results of the two-dimensional wound healing assay revealing nonsignificant migratory activity of MCF7 cells cultured on PAN/ G10 membranes. Potentially, the microenvironment of the electrically active graphenerich areas would preferentially attract electrically conductive proteins within the cell, as actin, avoiding their migration capacity towards more isolating graphene-poor areas.

Although the precise mechanism of graphene as a migrastatic agent is uncertain, the observed properties (particularly electrical) of PAN/G10 membranes led us to propose that graphene could affect cell migration through two mechanisms involving matrix metalloproteinase 9 (MMP-9): (i) downregulating the expression of MMP-9 through the 
inactivation of Rho GTPases and subsequent inhibition of cell polarity via local cellsubstrate mediated interactions, or (ii) inhibiting MMP-9 activation, i.e., by acting as an electron trap affecting the redox processes of MMP-9 (Hariono et al. 2018; Sancéau et al. 2003). Future studies are needed to further understand the antimigratory role of graphene as a potential migrastatic agent in cancer therapy.

\section{Conclusions}

Although the ability of graphene to induce spontaneous differentiation of stem cell types has been shown in previous works, as a major advancement, the results presented in this work remarkably revealed that the presence of graphene immobilized nonhomogeneously in PAN polymer membranes is able to induce changes in MCF7 breast tumor cells, resulting in both epithelial-like differentiation and a migrastatic effect without chemical agent-mediated signaling. Critical assessment of graphene-based membranes as antitumor and anti-invasion agents in cellular and animal models of cancer is warranted because they may provide new therapeutic strategies for cancer treatment. All elements appear to be in place for the incorporation of graphene-polymer composite substrates into the next steps of anticancer research.

\section{Methods}

\section{Preparation of PAN-graphene flat membranes}

PAN-graphene flat membranes were prepared by phase inversion reproducing the procedure described elsewhere (Diban et al. 2014, 2017). First, commercial multi-layered graphene (G) nanoplatelets (Av-PLAT-7, Avanzare, Spain) were dispersed by sonication during $20 \mathrm{~min}$ in the solvent $N$-methyl pyrrolidone (NMP, 99\% extrapure, Acros Organics, Madrid, Spain). Afterwards, PAN polymer (Mw, 150 kDa, J\&K Scientific GmbH, Madrid, Spain) was added in the G/NMP dispersion and stirred using a mechanical stirrer for $24 \mathrm{~h}$ until achieving a uniform PAN/G solution. The polymer solution was left to degasify overnight at room temperature. Subsequently, the polymer solution was casted on a glass plate casting knife with a space of $0.2 \mathrm{~mm}$ thickness and immediately submerged into a water/NMP coagulation bath $(80 / 20 \% \mathrm{v} / \mathrm{v})$. After complete polymer coagulation and removal of solvent traces by a washing procedure (Diban et al. 2014, 2017), the PAN/G flat membranes were stored in ultrapure water. For the characterization of the morphological, physicochemical and electrical properties, membrane samples were submerged in a $(50 / 50 \% \mathrm{v})$ glycerol/water bath before drying to preserve the membrane microstructure and then left to air dry.

Different polymer solution batches were prepared with 10\%wt of PAN and 0, 2, 10 and $15 \%$ wt of graphene (PAN/G0, PAN/G2, PAN/G10 and PAN/G15), respectively. For additional experimental procedures on PAN/G10 membrane synthesis see Additional file 9.

\section{Scanning electron microscopy}

The structure and morphology of the surface and cross section of the flat membranes were determined using scanning electron microscopy (SEM, EVO MA 15, Carl Zeiss) at a voltage of $20 \mathrm{kV}$. Cross section samples were frozen in liquid nitrogen for fracture. All 
the samples were kept overnight at $30{ }^{\circ} \mathrm{C}$ under vacuum and were gold sputtered before examination.

The fraction of porous area of the membranes (PF) was quantified analyzing at least three SEM images $(n \geq 3)$ from the surface and cross section using ImageJ (Fiji, U. S. National Institutes of Health, USA) software following the procedure explained by Buckman et al. (2017). Additionally, the thickness of the wetted PAN and PAN/G membranes, $\delta$, was measured using an electronic micrometer (Standard, Series 293, Mitutoyo) for $n \geq 4$ samples.

\section{Raman spectroscopy}

Raman spectroscopy of the PAN/G10 functionalized membranes was carried out by a Confocal-Raman NRS-4500 JASCO. A 532-nm wavelength beam from green diode laser was focused with a 50× MPlan FLN, 0.8 NA objective for detection, and an effective laser power of $4.4 \mathrm{~mW}$ was employed for all measurements. All analyses were performed from 1000 to $4400 \mathrm{~cm}^{-1}$ at $25 \%$ power with an exposition time of $10 \mathrm{~s}$ and 5 accumulations. A lattice of $144 \times 88 \mu \mathrm{m}$ was done in the PAN/G10 functionalized membrane with a space of $8 \mu \mathrm{m}$ between each point. Moreover, different discrete points were taken at two different altitudes $(Z=0$ and $Z=15)$ for the analysis of MCF7 cancer cells clusters on the PAN/G10 surface membrane. In this case, to avoid damage to the biological samples, a low incident laser power $(1.8 \mathrm{~mW}$ at $10 \%)$ with an exposition time of $5 \mathrm{~s}$ and 5 accumulations was used. These analyses were performed from 600 to $3800 \mathrm{~cm}^{-1}$. The analysis of the peaks and ratios were performed using Lorentzian functions with Origin Pro 2017 (OriginLab Corporation) and Micro Imaging Analysis (Spectra Manager Jasco) software.

\section{Contact angle measurements}

Contact angle was measured on dry membranes to determine wettability with water and DMEM culture medium using sessile-drop method; DSA25 (Drop Shape Analyzer, Krüss, Germany) with at least nine images $(n \geq 9)$.

\section{Electrical impedance}

Electrical conductivity and dielectric permittivity properties were evaluated along the thickness of the PAN/G2, PAN/G10 and PAN/G15 membranes via electrical impedance measurements on dry membrane samples similarly as described in (Sánchez-González et al. 2018). The experiments were carried out using a PM 6304 programmable automatic RCL meter (Philips) that was connected to two nickel foils acting as electrodes with a surface contact area $(S)$ of $5 \times 5 \mathrm{~cm}^{2}$. The dry membranes, used as electrolytes, were cut and located between the foils covering their contact area. The system was fixed with clamps during the measurements and the tests were carried out for at least two samples of each flat membrane $(n \geq 2)$ using a variation in the potential frequency $(f)$ in the range 50 to $100,000 \mathrm{~Hz}$ at room temperature. The equipment gives as a result the impedance modulus $(Z)$ and the phase angle $(\phi)$.

The resistivity of the membranes $(\rho, \Omega \mathrm{m})$ was calculated using Eq. (1):

$$
\rho=Z \cdot \cos \phi \cdot S / \delta
$$


where $S$ is the surface of the contact area $\left(\mathrm{m}^{2}\right)$ and $\delta$ is the thickness of the membranes $(\mathrm{m})$. While the dielectric permittivity $\left(\varepsilon_{r}\right)$ was obtained as follows:

$$
\varepsilon_{r}=\delta / 2 \pi f Z \cdot \operatorname{sen} \phi \cdot S
$$

Finally, the electrical conductivity $\left(\sigma, \mathrm{S} \mathrm{m}^{-1}\right)$ and the relative dielectric permittivity $\left(\varepsilon^{\prime}\right)$ were calculated as expressed in Eqs. (3) and (4), respectively, where $\varepsilon_{0}$ is the vacuum permittivity constant, $8.8510^{-12} \mathrm{Fm}^{-1}$ :

$$
\begin{aligned}
& \sigma=1 / \rho \\
& \varepsilon^{\prime}=\varepsilon_{r} / \varepsilon_{0}
\end{aligned}
$$

For additional experimental procedures on electrical impedance of PAN/G10 membranes see Additional file 9.

\section{Cell cultures}

The biocompatibility study of PAN (PAN/G0) and functionalized membranes with different concentrations of graphene (PAN/G) was carried out in the following cell lines: MCF7 (HTB-22 from ATCC: human adenocarcinoma from mammary gland cell line) and NSC34 (CLU140 from Cedarlane Laboratories: mouse motor neuron-like cell line). Cells were cultured in Dulbecco's Modified Eagle's Medium (DMEM) culture medium with high glucose content and without sodium pyruvate, enriched with $10 \%$ fetal bovine serum (FBS), $1 \%$ of non-essential amino acids (NEAA) and 1\% of penicillin/streptomy$\operatorname{cin}(\mathrm{P} / \mathrm{S})$ at $37{ }^{\circ} \mathrm{C}$ in a humid atmosphere containing $5 \% \mathrm{CO}_{2}$. Under normal conditions, cells were passed when they reached $80 \%$ of confluence and the medium was changed every 2-3 days. All culture media and supplements were acquired from Gibco (Thermo Fisher).

To prepare PAN membranes for cell culture, circular samples of $\varnothing 12 \mathrm{~mm}$ were cut from representative and homogenous sections of the as prepared PAN and PAN/G membranes. Using surgical silicone, they were glued to a $\varnothing 10 \mathrm{~mm}$ glass coverslip to prevent floating. Each sample was sterilized with $70 \% \mathrm{v} / \mathrm{v}$ EtOH in distilled water for $30 \mathrm{~min}$ and subsequent exposure to UV light for 15 min under a vertical laminar cabinet of biosecurity type II. Finally, the membranes were washed three times with sterile phosphate-buffered saline (PBS) to remove ethanol traces. To seed the cells on the membranes, $3 \times 10^{5}$ MCF7 or $5 \times 10^{5}$ NSC34 cells per $\mathrm{cm}^{2}$ were seeded uniformly onto the flat membranes. Cell culture plates were kept at $37^{\circ} \mathrm{C}$ in a humid atmosphere containing $5 \% \mathrm{CO}_{2}$ and allowed them to attach a minimum of $24 \mathrm{~h}$ before analysis.

For additional experimental procedures on cell culture see Additional file 9.

\section{TUNEL assay}

TUNEL staining was performed on cells grown on glass coverslips or PAN/G10 membranes using the FITC-conjugated in situ cell death detection kit (Roche) according to the manufacturer's instructions. Briefly, cells fixed in 3.7\% paraformaldehyde in PBS for $15 \mathrm{~min}$ at room temperature were incubated in the reagent (1:10 dilution of the enzyme solution (terminal deoxynucleotidyl transferase from calf thymus) in label solution 
(nucleotide mixture) in a humid chamber for $45 \mathrm{~min}$ at $37^{\circ} \mathrm{C}$. Samples were counterstained with DAPI and mounted using antifade medium Vectashield (VectorLabs). Fluorescence images were obtained with a fluorescence microscope (Axioskop 2 Plus, ZEISS) at $20 \times$ magnification coupled with a camera (Axiocam HRC). TUNEL-positive cells were quantified from a minimum of three images, containing at least 50 cells per image $(n=150)$, and the mean percentage of apoptotic cells was quantified.

\section{Wound healing assay}

The in vitro wound healing assay was performed using $35 \mathrm{~mm}$-dishes ( $\mu$-dish, IBIDI $\mathrm{GmbH})$. The presence of culture inserts allows cells to grow in two designated areas (each $0.22 \mathrm{~cm}^{2}$ ) with a predetermined gap size of $500 \pm 100 \mu \mathrm{m}$. To grow the cells over the two different substrates, PAN/G10 functionalized membranes and glass coverslips, the culture inserts were removed from the dishes, and the substrates were placed at the bottom of the IBIDI dishes. Inserts were pasted back by the outer edges over the different substrates (PAN/G10 and glass) using biocompatible surgical silicone. After sterilization with $75 \% \mathrm{Et}-\mathrm{OH}$ and UV irradiation, MCF7 stably expressing the Green Fluorescent Protein (GFP) $\left(6 \times 10^{5}\right.$ cells per dish) were seeded and incubated at $37^{\circ} \mathrm{C}$ in a humidified $5 \% \mathrm{CO}_{2}$ atmosphere until they reached confluency. At this point, culture inserts were removed with sterile forceps and one dish (0-h point) was processed for immunofluorescence and confocal microscopy. A second IBIDI dish was cultured for an additional 24-h period, to allow the cells to migrate over the cell-free gap or "wound".

The migration of the cells over the wound was observed by image capture at $\times 10$ magnification using a fluorescence microscope (Axioskop 2 Plus, ZEISS) coupled with a digital camera (Axiocam HRC). Images were analyzed using the ImageJ software, setting the correct scale for measuring the wound width closure $(\mu \mathrm{m})$, at the different time-points. Five images from each wound with 20 measurements per image were analyzed.

\section{Immunofluorescence and confocal microscopy}

Cell samples were fixed with freshly prepared 3.7\% paraformaldehyde in PBS for 15 min and permeabilized with Triton X-100 (0.5\% in PBS) for $15 \mathrm{~min}$, at room temperature. Samples were then incubated overnight at $4{ }^{\circ} \mathrm{C}$ with the following primary antibodies: mouse monoclonal anti-E-cadherin (dilution 1:100, BD Transduction Laboratories) and rabbit polyclonal anti-H3K9Ac (dilution 1:100 Upstate) or anti-H4Ac (dilution 1:100 Upstate). Next day, samples were washed with PBS and incubated for $45 \mathrm{~min}$ in the specific secondary antibody conjugated with Cy3 (1:750, Jackson ImmunoResearch Laboratories). Some samples were counterstained with Phalloidin-FITC conjugate (Sigma), which binds polymerized F-actin, used to identify cytoskeletal actin filaments, and with DAPI (4',6-diamidino-2-phenylindole). All samples were mounted with antifade medium Vectashield (VectorLabs) and confocal images were obtained with a LSM510 (Zeiss) laser scanning microscope using a $63 \times$ oil objective $(1.4 \mathrm{NA})$. In order to avoid overlapping signals, images were obtained by sequential excitation at $488 \mathrm{~nm}$ and $543 \mathrm{~nm}$, to detect FITC and Cy3, respectively. Images were then processed using Photoshop CC2019 software (Adobe).

For additional experimental procedures on live cell microscopy in real-time see Additional file 9 . 


\section{Transmission electron microscopy}

For cellular junctions' examination, samples PAN/G10 flat membranes with MCF7 cells grown over $48 \mathrm{~h}$ were fixed using $3 \%$ glutaraldehyde in $0.12 \mathrm{M}$ phosphate buffer $\left(0.12 \mathrm{M} \mathrm{Na}_{2} \mathrm{HPO}_{4}\right.$ and $\left.0.12 \mathrm{M} \mathrm{NaH}_{2} \mathrm{PO}_{4}\right)$ at $\mathrm{pH} 7.4$ for $2 \mathrm{~h}$, washed in $0.12 \mathrm{M}$ phosphate buffer three times and further fixed in $1 \% \mathrm{OsO}_{4}$ for $3 \mathrm{~h}$, washed again and dehydrated in increasing concentrations of acetones followed by propylene oxide and embedded Araldite (Electron Microscopy Sciences). Transversal Sections (500 $\mu \mathrm{m}$ thick) were obtained with a vibratome (Microm HM 650 V, Thermo Scientific) taking into account the presence of the flat membrane. Ultrathin sections mounted in copper grids were stained with uranyl acetate and lead citrate and examined with a JEOL-JEM 1011 transmission electron microscope operated at $80 \mathrm{kV}$.

\section{Western blotting}

MCF7 cells were scratched off from PAN/G10 flat membranes $(n=3)$ and control glass $(n=3)$ and resuspended in $1 \times$ Laemmli buffer (2\% SDS, 0.1\% 2-mercaptoethanol, $10 \%$ glycerol, $0.0005 \%$ bromophenol blue, and $63 \mathrm{mM}$ Tris- $\mathrm{HCl} \mathrm{pH}$ 6.8) supplemented with complete protease inhibitor cocktail. The samples were boiled for $10 \mathrm{~min}$ at $98{ }^{\circ} \mathrm{C}$ and cleared by centrifugation at $12,000 \mathrm{rpm}$ for $10 \mathrm{~min}$ at $4{ }^{\circ} \mathrm{C}$. The proteins were separated on 4-20\% Nu-Page TG polyacrylamide SDS-PAGE gels (Invitrogen), or 16\% acrylamide gels, optimal for histone protein analysis, and transferred to nitrocellulose membranes ( $0.2 \mu \mathrm{m}$ pore size, Life Technologies) by standard procedures. Following, membranes were rinsed twice with PBS with 0.1\% Tween-20 (PBS-T) and blocked with 5\% non-fat dried milk (Biorad) in PBS-T. Then, membranes were incubated with primary antibodies; rabbit polyclonal antibodies anti-E-cadherin (Cell Signaling Technology, dilution 1:1000), anti-H3K9Ac (Upstate, Cat. 06599, dilution 1:1000), anti-H4Ac (Upstate, Cat. 06598, dilution 1:1000), anti-Histone H3 (Pierce, dilution 1:5000) and mouse monoclonal antibody to GAPDH (Chemicon Intl., Temecula, dilution 1:5000) diluted in 0.5\% BSA in PBS-T overnight at $4{ }^{\circ} \mathrm{C}$. Afterwards, membranes were incubated with specific anti-rabbit IRDye680DX (Rockland Immunochemicals, USA) secondary antibodies diluted 1:5000 in PBS-T for $1 \mathrm{~h}$ at room temperature. Protein bands were detected with an Odyssey ${ }^{\mathrm{TM}}$ Infrared-Imaging System (Li-Cor Biosciences) according to Odyssey ${ }^{\mathrm{TM}}$ Western-Blotting Protocol. For the quantitative analysis of the blots ImageJ software was used.

\section{Real-time quantitative PCR for relative gene expression analysis}

MCF7 cells cultured onto the developed scaffolds (PAN/G10 group) or on culture plates (control group) for $48 \mathrm{~h}(n=3)$ were used for RT-qPCR studies. RNA was extracted cell cultures using Trizol (Invitrogen, Carlsbad) reagent and purified with the RNeasy kit (Qiagen, Hilden) according to the manufacturer's instructions and quantified using a NanoDrop ND-1000 spectrophotometer (Nanodrop Technologies). One microgram of RNA was reverse-transcribed to first-strand cDNA using a High-Capacity cDNA Reverse Transcription Kit (Life Technologies) using random hexamers as primers. The expression of ID1, CDH1, EPCAM, KRT18, ZO1, ACTB and CTNNB1 mRNAs were determined by RT-qPCR using gene-specific SYBR Green-based primers (Thermo). Each individual RT-qPCR assay was done in technical triplicates. The relative expression 
levels of target genes were calculated using the comparative $2^{-\triangle \Delta C t}$ method after normalization using GAPDH as an endogenous control, as no difference in GAPDH mRNA expression between MCF7 cells grown on Petri dishes (Ctrl) and PAN/G10 (PAN) was observed. The DNA sequence of the primers used in this study were as follows: GAPDH forward 5'-ACAACTTTGGTATCGTGGAAGG-3', GAPDH reverse 5'-GCCATCACG CCACAGTTTC-3', ID1 forward 5'-AATCCGAAGTTGGAACCCCC-3', ID1 reverse 5'-ACACAAGATGCGATCGTCCG-3', CDH1 forward 5'-GCCTCCTGAAAAGAG AGTGGAAG-3', CDH1 reverse 5'-TGGCAGT GTCTCTCCAAATCCG-3', EPCAM forward 5'-GCCAGTGTACTTCA GTTGGTGC-3', EPCAM reverse 5'-CCCTTCAGG TTTTGCTCTTCTCC-3', KRT18 forward 5'-GCTGGAAGATGGCGAGGACTTT-3', KRT18 reverse 5'-TGGTCTCAGACACCACTTTGCC-3', CTNNB1 forward 5'-CAC AAGCAGAGTGCTGAAGGTG-3', CTNNB1 reverse 5'-GATTCCTGAGAGTCCAA AGACAG-3', ZO1 forward 5'-GTCCAGAATCTCGGAAAAGTGCC-3', ZO1 reverse 5'-CTTTCAGCGCACCATACCAACC-3', ACTB forward 5'-CACCATTGGCAATGA GCGGTTC-3' and $A C T B$ reverse 5'-AGGTCTTTGCGGATGTCCACGT-3'.

\section{Methylation-specific polymerase chain reaction (MSP) Genomic DNA (gDNA) extraction}

MCF7 cells grown in 35-mm petri dishes or over PAN/G10 membranes were trypsinized and resuspended in $160 \mu \mathrm{L}$ of PBS. gDNA was purified using the ReliaPrep gDNA Tissue Miniprep System (Promega) following manufacturer's instructions. Briefly, cell suspensions and tissue fragments from a human control dorsal root ganglion were incubated at $56{ }^{\circ} \mathrm{C}$ with $200 \mu \mathrm{L}$ cell lysis buffer and $20 \mu \mathrm{L}$ proteinase $\mathrm{K}$ solution $(10 \mathrm{mg} / \mathrm{mL})$ for $30 \mathrm{~min}$. After an additional $10 \mathrm{~min}$ incubation step, at $56^{\circ} \mathrm{C}$ with RNAse A solution, gDNA was precipitated. Using the ReliaPrep binding columns, gDNA was washed 3 times, eluted in $30 \mu \mathrm{L}$ of nuclease-free water, quantified using a Nano-drop spectrophotometer and stored at $-80^{\circ} \mathrm{C}$ until use.

\section{Sodium bisulfite treatment}

The principle of DNA modification with the sodium bisulfite technique is based on its ability to convert all nonmethylated cytosine residues into uracils through deamination whereas the methylated cytosine is resistant to the reaction and remains as cytosine. The methylation specific PCR (MSP) design exploits these differences to discriminate between the methylated and nonmethylated sequences. gDNA was bisulfite treated using EZ DNA Methylation-Gold Kit (Zymo), that ensures a conversion efficiency of at least $99 \%$ of non-methylated $C$ residues to $U$ and a $99 \%$ protection of methylated cytosines, according to manufacturer's instructions. Briefly, $500 \mathrm{ng}$ of gDNA from MCF7 cell grown on either Petri dishes (MCF7-Ctrl), or on PAN/G10 (MCF7-PAN) and from a normal non-tumoral tissue (MSP-control $(+)$ ) were diluted in $20 \mu \mathrm{L}$ of water and combined with $130 \mu \mathrm{L}$ of freshly prepared CT conversion reagent. The bisulfite DNA conversion was performed using the following conditions: denaturation $10 \min 98^{\circ} \mathrm{C}$, incubation $150 \mathrm{~min} 64{ }^{\circ} \mathrm{C}$, hold $4{ }^{\circ} \mathrm{C}$. The bisulfite converted DNA was purified following manufacturer's instructions. Briefly, the bisulfite reaction was mixed with $600 \mu \mathrm{L}$ of binding buffer, applied to the spin column and centrifuged at 12,000 rpm for $1 \mathrm{~min}$. The flow through was discarded and the column washed with $100 \mu \mathrm{L}$ of wash buffer 
(WB). Desulphonation buffer $(200 \mu \mathrm{L})$ was applied to the column and incubated at room temperature for $20 \mathrm{~min}$. The column was centrifuged and then washed twice with WB $(200 \mu \mathrm{L})$. Residual BW buffer was removed by an additional spin (12,000 rpm, $1 \mathrm{~min}$ ). Nuclease-free water $(10 \mu \mathrm{L})$ was added to the column to elute the DNA and stored at $-80^{\circ} \mathrm{C}$ until use. Modified DNA $(1 \mu \mathrm{L})$ was PCR amplified with two previously designed pairs of methylation-specific PCR (MSP) primers for the promoter region of the $C D H 1$ gene (Sasaki et al. 2003). The MSP primers for methylated DNA were MSP-metCDH1 for (5'-TTAGGTTAGAGGGTTATCGCGT-3) and MSP-metCDH1 rev (5'-AAATAA ACCCCGAAACACCG-3), being the PCR product size $116 \mathrm{bp}$. The PCR conditions were $94{ }^{\circ} \mathrm{C}$ for $3 \mathrm{~min} ; 35$ cycles of $94{ }^{\circ} \mathrm{C}$ for $30 \mathrm{~s}, 57^{\circ} \mathrm{C}$ for $30 \mathrm{~s}$, and $72{ }^{\circ} \mathrm{C}$ for $45 \mathrm{~s}$; and a final extension at $72{ }^{\circ} \mathrm{C}$ for $5 \mathrm{~min}$. For unmethylated DNA amplification, the following MSP primers were used MSP-unmetCDH1 for (5'-TAATTTTAGGTTAGAGGG TTATTGT-3) and MSP-unmetCDH1 rev (5'-CACAACCAATCAACAACACA-3). For the unmethylated pair of primers, the product size was $97 \mathrm{bp}$. The PCR conditions were $94{ }^{\circ} \mathrm{C}$ for $3 \mathrm{~min} ; 35$ rounds of $94{ }^{\circ} \mathrm{C}$ for $30 \mathrm{~s}, 53{ }^{\circ} \mathrm{C}$ for $30 \mathrm{~s}$, and $72{ }^{\circ} \mathrm{C}$ for $45 \mathrm{~s}$; and $72{ }^{\circ} \mathrm{C}$ extension for $5 \mathrm{~min}$. The PCR products were separated by electrophoresis through a $2 \%$ agarose gel containing $0.01 \%$ RedSafe. DNA bands were visualized by ultraviolet light, digitized using GelDoc (Biorad) and quantified using ImageJ (NIH).

\section{Quantification, morphometric measurements and statistical analysis}

Quantitative cellular experiments were run in triplicate for each cell line and substrate. Adhesion capacity (d0) of cells to adhere into PAN and PAN/G flat membranes was evaluated on DAPI-counterstained cells using an epifluorescence light microscope (AxiosKop 2 plus, Zeiss) coupled with an AxioCam HRc (Zeiss) camera. The total number of nuclei cells per unit of area was counted from at least 3 micrographs for adhesion. Furthermore, morphological analysis on Phalloidin-counterstained MCF7 cells was performed to compare cell morphology $24 \mathrm{~h}$ after the seeding on control glass coverslips or PAN/G10 flat membranes. Using the ImageJ software, the value of the cell area and the circularity shape factor were obtained. The circularity shape factor is defined as the degree to which the particle is like a circle, taking into consideration the smoothness of the perimeter $\left(C=\sqrt{ }\left(4 \cdot \pi \cdot A / P^{2}\right)\right)$ (Olson 2011). This factor varies between 0 and 1 depending on whether the cell shape is rounded (value close to 1 ) or fusiform (value close to 0). For the densitometric analysis of H3K9ac and H4ac fluorescence signal, 3 images with more than 100 cells per image were used. Counterstaining with DAPI allowed to adjust the threshold and add nuclear areas to the Image)'s ROI (region of interest) manager. Each ROI was used to measure the total amount of fluorescence signal within each ROI to latter normalized to the corresponding nuclear area.

All results are expressed as mean \pm standard deviation, unless otherwise stated, and data were statistically analyzed with the unpaired two-tailed Student's $t$ test to determine differences among the two groups using GraphPad Prism (GraphPad Software) and Microsoft Excel software packages (Microsoft). The statistical significance was considered at $p<0.05$.

\section{Supplementary Information}

The online version contains supplementary material available at https://doi.org/10.1186/s12645-021-00107-6. 
Additional file 1: Figure S1. (A) Culture medium contact angle average values of the indicated PAN/G membranes. (B, C) Plots of the relative dielectric permittivity (B) and electric conductivity (C) of the PAN/G2; PAN/G10 and PAN/ G15 membranes measured by means of electrical impedance between $10^{3}$ and $10^{4} \mathrm{~Hz}$ of applied frequency.

Additional file 2: Figure S2. (A) Dot plot comparing the $I_{\mathrm{CN}} / I_{\mathrm{G}}$ ratio from Raman spectra of batch 1 (uneven $\mathrm{G}$, yellow dots) and batch 2 (even G, green dots) PAN/G10 membranes after a surface mapping of $0.144 \times 0.088 \mathrm{~mm}$, where a minimum of 30 points were analyzed. Line on each dot plot represents the mean. (B, D) Optical images of the surface of batch 1 PAN/G10 membrane (uneven $G, B$ ) and batch 2 (even G, D). (C, E) Confocal images of MCF7 cells after 2 days of culture on PAN/G10 membranes of batch 1 (uneven $G, C$ ) and batch 2 (even $G, E$ ). ***: $p<0.0005$. Scale bar: $50 \mu \mathrm{m}$ (C and E).

Additional file 3: Figure S3. Fluorescence microscopy images of MCF7 (A-D) and NSC34 (F-I) cell nuclei stained with DAPI, a cytochemical marker of DNA. Both cell types were grown on glass coverslips ( $A$ and F) or on PAN membranes with increasing amounts $0 \%, 2 \%$ and $10 \%$ of graphene (PAN/G0, B, G; PAN/G2, C, H; PAN/G10, D, I). (E and J) Total cell number per area unit was calculated from at least three different images taken from MCF7 and NSC34 cell cultures, respectively. Bars represent mean + SD. ${ }^{*}: p<0.05,{ }^{* *}: p<0.005,{ }^{* * *}: p<0.0005$. Scale bar: $50 \mu \mathrm{m}(\mathrm{A}-1)$.

Additional file 4: Figure S4. (A-H) Confocal microscopy images of Phalloidin-FITC stained SH-SY5Y (A, B), U87 (C-D), C6 $(E, F)$ and HeLa $(G, H)$ cell lines derived from different cancer types (neuroblastoma, glioblastoma, glioma and cervix carcinoma) with a well-known migratory capacity were grown for 24-48 h, except for SH-SY5Y (5 days), on glass coverslips or PAN/G10 as indicated. (I-L) SV40 and hTERT immortalized non-tumoral MEFs and ASC52 cell cultures were also analyzed by confocal microscopy. Note in $\mathrm{J}$ and $\mathrm{L}$ that the morphology of non-carcinogenic cells is not

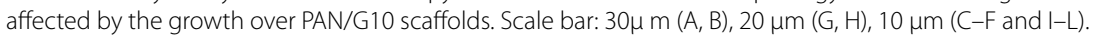

Additional file 5: Figure S5. (A) Three-dimensional reconstruction of confocal microscope images of a MCF7 cellular islet formed after growth over PAN/G10 for 5 days and co-stained with Phalloidin-FITC and DAPI. (B) Image was rotated $90^{\circ}$ from the previous (top to bottom) to observe the vertical growth of the islet. (C) Gallery of the images used for 3D-reconstruction in (A). Note that all cross-sectional views of the islet show compact cellular aggregation. Scale bar: $30 \mu \mathrm{m}$.

Additional file 6: Video S1. Three-dimensional reconstruction video from Z-stack series of confocal microscope images taken from a typical MCF7 multicellular islet formed after growth over PAN/G10 for 5 days.

Additional file 7: Video S2. 18-h time-lapse confocal imaging of GFP-expressing MCF7 cells grown on 35 um-glass bottom IBIDI dish.

Additional file 8: Video S3. 18-h time-lapse confocal imaging of GFP-expressing MCF7 cells grown on PAN/G10 membranes. Note how MCF7 cells grown on PAN/G10 form cell islets that have the capacity to sequester near-by cells abolishing their migratory capacity.

Additional file 9. Additional experimental procedures.

\section{Acknowledgements}

The authors would like to thank Prof. Francisco Vicente, Dr. José Juan García Jareño, and Dr. Jeronimo Agrisuelas of the Department of Physical Chemistry of the University of Valencia (Spain), Dr. Sandra Sánchez-González for the measurements of electrical impedance. We also wish to thank for their technical assistance to Raquel García-Ceballos of the Department of Anatomy and Cell Biology (UC), Fidel Madrazo of the Advanced Microscopy Facility (IDIVAL) and Alvaro del Real and Carolina Sañudo of the Department of Internal Medicine (HUMV-IDIVAL-UC). MM-O acknowledges the FPU Grant (19/02324) awarded by the Spanish Ministry of Science and Innovation.

\section{Authors' contributions}

$\mathrm{ND}, \mathrm{MM}-\mathrm{O}, \mathrm{AP}-\mathrm{B}$ and $\mathrm{OT}$ designed and performed experiments. ML and MB contributed to data compilation and paper preparation. MJR and AU provided critical feedback on the study and contributed to the preparation of the paper. ND and OT devised the study and oversaw the research program. All authors listed reviewed the paper and provided feedback. All authors read and approved the final manuscript.

\section{Funding}

This work was supported by Grants from the "Asociación Luchamos por la Vida" (Corrales de Buelna, Cantabria, Spain), Health Research Institute "Valdecilla" (INNVAL17/20, IDIVAL), MINECO/EIG-Concert Japan (X-MEM PCI2018-092929 project, International Joint Program 2018) and the Spanish Research Agency (PID2019-105827RB-100 supported by MCIN/ AEI/10.13039/501100011033).

\section{Declarations}

Ethics approval and consent to participate

Not applicable.

\section{Consent for publication}

All authors consent to publish.

\section{Competing interests}

The authors declare that they have no known competing financial interests or personal relationships that could have appeared to influence the work reported in this paper. 


\section{Availability of data and materials}

The authors declare that all other relevant data supporting the findings of the study are available in this article and in its additional information file. Access to our raw data can be obtained from the corresponding author upon reasonable request.

\section{Author details}

${ }^{1}$ Department of Chemical and Biomolecular Engineering, ETSIIyT, University of Cantabria, Santander, Spain. ${ }^{2}$ Health Research Institute "Valdecilla" (IDIVAL), Santander, Spain. " "Centro de Investigación Biomédica en Red Sobre Enfermedades Neurodegenerativas" (CIBERNED), Madrid, Spain. ${ }^{4}$ Department of Molecular Biology, University of Cantabria, Santander, Spain. ${ }^{5}$ Department of Physiology and Pharmacology, University of Cantabria, Santander, Spain. ${ }^{6}$ Department of Anatomy and Cell Biology, University of Cantabria, Santander, Spain. " Universidad Europea del Atlántico", Santander, Spain.

Received: 26 July 2021 Accepted: 25 November 2021

Published online: 04 January 2022

\section{References}

Abramczyk H, Surmacki J, Kopeć M, Olejnik AK, Lubecka-Pietruszewska K, Fabianowska-Majewska K (2015) The role of lipid droplets and adipocytes in cancer. Raman imaging of cell cultures: MCF10A, MCF7, and MDA-MB-231 compared to adipocytes in cancerous human breast tissue. Analyst 140(7):2224-2235

Andrews JL, Kim AC, Hens JR (2012) The role and function of cadherins in the mammary gland. Breast Cancer Res 14(1):1-10

Bajaj P, Rivera JA, Marchwiany D, Solovyeva V, Bashir R (2014) Graphene-based patterning and differentiation of C2C12 myoblasts. Adv Healthc Mater 3(7):995-1000

Baranwal S, Alahari SK. Molecular mechanisms controlling E-cadherin expression in breast cancer. Biochem Biophys Res Commun. 2009;384(1):6-11. https://www2.tri-kobe.org/nccn/guideline/breast/english/breast.pdf

Brooks SC, Locke ER, Soule HD (1973) Estrogen receptor in a human cell line (MCF 7) from breast carcinoma. J Biol Chem 248(17):6251-6253

Buckman J, Bankole SA, Zihms S, Lewis H, Couples G, Corbett PWM (2017) Quantifying porosity through automated image collection and batch image processing: case study of three carbonates and an aragonite cemented sandstone. Geoscience 7(3):70

Caldeira JRF, Prando ÉC, Quevedo FC, Moraes Neto FA, Rainho CA, Rogatto SR (2006) CDH1 promoter hypermethylation and $\mathrm{E}$-cadherin protein expression in infiltrating breast cancer. BMC Cancer 6:1-9

Canel M, Serrels A, Frame MC, Brunton VG (2013) E-cadherin-integrin crosstalk in cancer invasion and metastasis. J Cell Sci 126(2):393-401

Carré A, Lacarrière V (2010) How substrate properties control cell adhesion. A physical-chemical approach. J Adhes Sci Technol 24(5):815-830

Charles-Harris M, Navarro M, Engel E, Aparicio C, Ginebra MP, Planell JA (2005) Surface characterization of completely degradable composite scaffolds. J Mater Sci Mater Med 16(12):1125-1130

Chen T, Li M, Liu J (2018) $\pi-\pi$ stacking interaction: a nondestructive and facile means in material engineering for bioapplications. Cryst Growth Des 18(5):2765-2783

Clayton AL, Hazzalin CA, Mahadevan LC (2006) Enhanced histone acetylation and transcription: a dynamic perspective. Mol Cell 23(3):289-296

Comşa Ş, Cîmpean AM, Raica M (2015) The story of MCF-7 breast cancer cell line: 40 years of experience in research. Anticancer Res 35(6):3147-3154

Corso G, Figueiredo J, De Angelis SP, Corso F, Girardi A, Pereira J et al (2020) E-cadherin deregulation in breast cancer. J Cell Mol Med 24(11):5930-5936

De Craene B, Berx G (2013) Regulatory networks defining EMT during cancer initiation and progression. Nat Rev Cancer 13(2):97-110. https://doi.org/10.1038/nrc3447

De Vitis S, Coluccio ML, Gentile F, Malara N, Perozziello G, Dattola E et al (2016) Surface enhanced Raman spectroscopy measurements of MCF7 cells adhesion in confined micro-environments. Opt Lasers Eng 76:9-16. https://doi.org/10. 1016/j.optlaseng.2015.04.010

Diban N, Ramos-Vivas J, Remuzgo-Martinez S, Ortiz I, Urtiaga A (2014) Poly( $\mathcal{E}$-caprolactone) films with favourable properties for neural cell growth. Curr Top Med Chem 14(23):2743-2749

Diban N, Sánchez-González S, Lázaro-Díez M, Ramos-Vivas J, Urtiaga A (2017) Facile fabrication of poly(ع-caprolactone)/ graphene oxide membranes for bioreactors in tissue engineering. J Membr Sci 540:219-228

Ding W, Dang H, You H, Steinway S, Takahashi Y, Wang HG et al (2012) MiR-200b restoration and DNA methyltransferase inhibitor block lung metastasis of mesenchymal-phenotype hepatocellular carcinoma. Oncogenesis 1(6):e15-e11 https://doi.org/10.1038/oncsis.2012.15

Downing TL, Soto J, Morez C, Houssin T, Fritz A, Yuan F et al (2013) Biophysical regulation of epigenetic state and cell reprogramming. Nat Mater 12(12):1154-1162. https://doi.org/10.1038/nmat3777

Draux F, Gobinet C, Sulé-Suso J, Trussardi A, Manfait M, Jeannesson P et al (2010) Raman spectral imaging of single cancer cells: probing the impact of sample fixation methods. Anal Bioanal Chem 397(7):2727-2737

Droufakou S, Deshmane V, Roylance R, Hanby A, Tomlinson I, Hart IR (2001) Multiple ways of silencing E-cadherin gene expression in lobular carcinoma of the breast. Int J Cancer 92(3):404-408

El Khal H, Cordier A, Batis N, Siebert E, Georges S, Steil MC (2017) Effect of porosity on the electrical conductivity of LAMOX materials. Solid State lonics 304:75-84. https://doi.org/10.1016/j.ssi.2017.03.028

Ferrari A, Cecchini M, Dhawan A, Micera S, Tonazzini I, Stabile R et al (2011) Nanotopographic control of neuronal polarity. Nano Lett 11(2):505-511 
Gandalovičová A, Rosel D, Fernandes M, Veselý P, Heneberg P, Čermák V et al (2017) Migrastatics_anti-metastatic and anti-invasion drugs: promises and challenges. Trends Cancer 3(6):391-406

Gharooni M, Alikhani A, Moghtaderi H, Abiri H, Mashaghi A, Abbasvandi F et al (2019) Bioelectronics of the cellular cytoskeleton: monitoring cytoskeletal conductance variation for sensing drug resistance. ACS Sens 4(2):353-362

Green KJ, Getsios S, Troyanovsky S, Godsel LM (2010) Intercellular junction assembly, dynamics, and homeostasis. Cold Spring Harb Perspect Biol 2(2):1-22

Gullekson C, Lucas L, Hewitt K, Kreplak L (2011) Surface-sensitive Raman spectroscopy of collagen I fibrils. Biophys 」 100(7):1837-1845. https://doi.org/10.1016/j.bpj.2011.02.026

Gulliford T, English J, Colston KW, Menday P, Moller S, Coombes RC (1998) A phase I study of the vitamin D analogue EB 1089 in patients with advanced breast and colorectal cancer. Br J Cancer 78(1):6-13

Gumbiner BM (1996) Cell adhesion: the molecular basis of tissue architecture and morphogenesis. Cell 84(3):345-357

Guo P, Chen W, Li H, Li M, Li L (2018) The histone acetylation modifications of breast cancer and their therapeutic implications. Pathol Oncol Res Pathol Oncol Res 24(4):807-813

Hanahan D, Weinberg RA (2011) Hallmarks of cancer: the next generation. Cell 144(5):646-674

Hariono M, Yuliani SH, Istyastono EP, Riswanto FDO, Adhipandito CF (2018) Matrix metalloproteinase 9 (MMP9) in wound healing of diabetic foot ulcer: molecular target and structure-based drug design. Wound Med 22(May):1-13. https:// doi.org/10.1016/j.wndm.2018.05.003

Jawaid K, Crane SR, Nowers JL, Lacey M, Whitehead SA (2010) Long-term genistein treatment of MCF-7 cells decreases acetylated histone 3 expression and alters growth responses to mitogens and histone deacetylase inhibitors. J Steroid Biochem Mol Biol 120(4-5):164-171. https://doi.org/10.1016/j.jsbmb.2010.04.007

Jung H, Yoon SR, Lim J, Cho HJ, Lee HG (2020) Dysregulation of rho GTPases in human cancers. Cancers (basel) 12(5):1-17

Kim J, Kim YR, Kim Y, Lim KT, Seonwoo H, Park S et al (2013) Graphene-incorporated chitosan substrata for adhesion and differentiation of human mesenchymal stem cells. J Mater Chem B 1(7):933-938

Kim SE, Kim MS, Shin YC, Eom SU, Lee JH, Shin DM et al (2016) Cell migration according to shape of graphene oxide micropatterns. Micromachines 7(10):1-10

Kim K, Nam KH, Lee J, Kim HJ, Goh M, Ku BC et al (2017) Effect of defect-healing in graphene nanosheets on the mechanical properties of polyimide nanocomposites. Carbon NY 122:614-621. https://doi.org/10.1016/j.carbon.2017.06.102

Kümper S, Marshall CJ (2011) ROCK-driven actomyosin contractility induces tissue stiffness and tumor growth. Cancer Cell 19(6):695-697

Lustberg MB, Ramaswamy B (2011) Epigenetic therapy in breast cancer. Curr Breast Cancer Rep 3(1):34-43

Manciu FS, Ciubuc JD, Parra K, Manciu M, Bennet KE, Valenzuela P et al (2017) Label-free Raman imaging to monitor breast tumor signatures. Technol Cancer Res Treat 16(4):461-469

Mantecón-Oria M, Diban N, Berciano MT, Rivero MJ, David O, Lafarga M et al (2020) Hollow fiber membranes of PCL and PCL/graphene as scaffolds with potential to develop in vitro blood-brain barrier models. Membranes (basel) 10(8):1-18

Martelli C, King A, Simon T, Giamas G (2020) Graphene-induced transdifferentiation of cancer stem cells as a therapeutic strategy against glioblastoma. ACS Biomater Sci Eng 6(6):3258-3269

Masi I, Caprara V, Bagnato A, Rosanò L (2020) Tumor cellular and microenvironmental cues controlling invadopodia formation. Front Cell Dev Biol 8(October):1036

Misra R, Kandoi S, Varadaraj S, Vijayalakshmi S, Nanda A, Verma RS (2020) Nanotheranostics: a tactic for cancer stem cells prognosis and management. J Drug Deliv Sci Technol 55(December 2019):101457. https://doi.org/10.1016/j.jddst. 2019.101457

Moll R, Mitze M, Frixen UH, Birchmeier W (1993) Differential loss of E-cadherin expression in infiltrating ductal and lobular breast carcinomas. Am J Pathol 143(6):1731-1742

Nass SJ, Herman JG, Gabrielson E, Iversen PW, Parl FF, Davidson NE et al (2000) Aberrant methylation of the estrogen receptor and E-cadherin $5^{\prime} \mathrm{CpG}$ islands increases with malignant progression in human breast cancer. Cancer Res 60(16):4346-4348

Olson E. Paricle shape factors and their use in image analysis_-part 1: theory as published in GXP, Summer 2011 (Vol15/ No3). 2011;15(1).

Onder TT, Gupta PB, Mani SA, Yang J, Lander ES, Weinberg RA (2008) Loss of E-cadherin promotes metastasis via multiple downstream transcriptional pathways. Cancer Res 68(10):3645-3654

Pálmer HG, González-Sancho JM, Espada J, Berciano MT, Puig I, Baulida J et al (2001) Vitamin D3 promotes the differentiation of colon carcinoma cells by the induction of E-cadherin and the inhibition of $\beta$-catenin signaling. J Cell Biol 154(2):369-387

Pijuan J, Barceló C, Moreno DF, Maiques O, Sisó P, Marti RM et al (2019) In vitro cell migration, invasion, and adhesion assays: from cell imaging to data analysis. Front Cell Dev Biol 7(JUN):1-16

Prydatko AV, Belyaeva LA, Jiang L, Lima LMC, Schneider GF (2018) Contact angle measurement of free-standing squaremillimeter single-layer graphene. Nat Commun 9(1):4185. https://doi.org/10.1038/s41467-018-06608-0

Ramirez Moreno M, Stempor PA, Bulgakova NA (2021) Interactions and feedbacks in E-cadherin transcriptional regulation. Front Cell Dev Biol 9(June):1-10

Romay M, Diban N, Rivero MJ, Urtiaga A, Ortiz I (2020) Critical issues and guidelines to improve the performance of photocatalytic polymeric membranes. Catalysts 10:570. https://doi.org/10.3390/catal10050570

Sadikovic B, Andrews J, Carter D, Robinson J, Rodenhiser DI (2008) Genome-wide H3K9 histone acetylation profiles are altered in benzopyrene-treated MCF7 breast cancer cells. J Biol Chem 283(7):4051-4060. https://doi.org/10.1074/ jbc.M707506200

Saleem J, Wang L, Chen C (2018) Carbon-based nanomaterials for cancer therapy via targeting tumor microenvironment. Adv Healthc Mater 7(20):1-30

Sancéau J, Truchet S, Bauvois B (2003) Matrix metalloproteinase-9 silencing by RNA interference triggers the migratoryadhesive switch in Ewing's sarcoma cells. J Biol Chem 278(38):36537-36546

Sánchez-González S, Diban N, Bianchi F, Ye H, Urtiaga A (2018) Evidences of the effect of GO and rGO in PCL membranes on the differentiation and maturation of human neural progenitor cells. Macromol Biosci 18(11):1-8 
Sanz-Moreno V, Gaggioli C, Yeo M, Albrengues J, Wallberg F, Viros A et al (2011) ROCK and JAK1 signaling cooperate to control actomyosin contractility in tumor cells and stroma. Cancer Cell 20(2):229-245

Sarafis P, Nassiopoulou AG (2014) Dielectric properties of porous silicon for use as a substrate for the on-chip integration of millimeter-wave devices in the frequency range 140 to $210 \mathrm{GHz}$. Nanoscale Res Lett 9(1):1-8

Sasaki M, Anast J, Bassett W, Kawakami T, Sakuragi N, Dahiya R (2003) Bisulfite conversion-specific and methylationspecific PCR: a sensitive technique for accurate evaluation of $\mathrm{CpG}$ methylation. Biochem Biophys Res Commun 309(2):305-309

Sleeman J, Steeg PS (2010) Cancer metastasis as a therapeutic target. Eur J Cancer 46(7):1177-1180

Stankic M, Pavlovic S, Chin Y, Brogi E, Padua D, Norton L et al (2013) TGF- $\beta$-Id1 signaling opposes twist1 and promotes metastatic colonization via a mesenchymal-to-epithelial transition. Cell Rep 5(5):1228-1242

Surowiec AJ, Stuchly SS, Barr JR, Swarup A (1988) Dielectric properties of breast carcinoma and the surrounding tissues. IEEE Trans Biomed Eng 35(4):257-263

Tycko B (2000) Epigenetic gene silencing in cancer. J Clin Invest 105(4):401-407

Vantangoli MM, Madnick SJ, Huse SM, Weston P, Boekelheide K (2015) MCF-7 human breast cancer cells form differentiated microtissues in scaffold-free hydrogels. PLOS ONE 10(8):1-20

Venza M, Visalli M, Catalano T, Biondo C, Beninati C, Teti D et al (2016) DNA methylation-induced E-cadherin silencing is correlated with the clinicopathological features of melanoma. Oncol Rep 35(4):2451-2460

Vesuna F, van Diest P, Chen JH, Raman V (2008) Twist is a transcriptional repressor of E-cadherin gene expression in breast cancer. Biochem Biophys Res Commun 367(2):235-241

Wang Y, Li Y, Huang J, Zhang Y, Ma R, Zhang S et al (2021) Correlation between electrical characteristics and biomarkers in breast cancer cells. Sci Rep 11(1):1-11. https://doi.org/10.1038/s41598-021-93793-6

Weinberg RA (2014) The biology of cancer, 2nd edn. Garland Science, Taylor \& Francis group, New York

Xia X, Wang Y, Zhong Z, Weng GJ (2017) A frequency-dependent theory of electrical conductivity and dielectric permittivity for graphene-polymer nanocomposites. Carbon NY 111:221-230. https://doi.org/10.1016/j.carbon.2016.09. 078

Yan X, Itoh T, Kitahama Y, Suzuki T, Sato H, Miyake T et al (2012) A Raman spectroscopy study on single-wall carbon nanotube/polystyrene nanocomposites: mechanical compression transferred from the polymer to single-wall carbon nanotubes. J Phys Chem C 116(33):17897-17903

Yoo J, Kim J, Baek S, Park Y, Im H, Kim J (2014) Cell reprogramming into the pluripotent state using graphene based substrates. Biomaterials 35(29):8321-8329. https://doi.org/10.1016/j.biomaterials.2014.05.096

Zhang X, Zhang Y, LiY (2013) ß-elemene decreases cell invasion by upregulating E-cadherin expression in MCF-7 human breast cancer cells. Oncol Rep 30(2):745-750

Zhou H, Zhang B, Zheng J, Yu M, Zhou T, Zhao K et al (2014) The inhibition of migration and invasion of cancer cells by graphene via the impairment of mitochondrial respiration. Biomaterials 35(5):1597-1607

Zhuang J, Huo Q, Yang F, Xie N (2020) Perspectives on the role of histone modification in breast cancer progression and the advanced technological tools to study epigenetic determinants of metastasis. Front Genet 11(October):1-15

\section{Publisher's Note}

Springer Nature remains neutral with regard to jurisdictional claims in published maps and institutional affiliations.

- fast, convenient online submission

- thorough peer review by experienced researchers in your field

- rapid publication on acceptance

- support for research data, including large and complex data types

- gold Open Access which fosters wider collaboration and increased citations

- maximum visibility for your research: over $100 \mathrm{M}$ website views per year

At BMC, research is always in progress.

Learn more biomedcentral.com/submissions 